\title{
Genomic hallmarks of cellular dormancy in cancer and therapeutic implications
}

Anna J. Wiecek ${ }^{1}$, Stephen J. Cutty ${ }^{2}$, Daniel Kornai ${ }^{1}$, Mario Parreno-Centeno ${ }^{1}$, Lucie E. Gourmet ${ }^{1}$, Guidantonio Malagoli Tagliazucchi ${ }^{1}$, Daniel H. Jacobson ${ }^{1,3}$, Ping Zhang ${ }^{4}$, Lingyun Xiong ${ }^{4}$, Gareth L. Bond ${ }^{5}$, Alexis R. Barr ${ }^{2,6}$, Maria Secrier ${ }^{1, *}$

${ }^{1}$ UCL Genetics Institute, Department of Genetics, Evolution and Environment, University College London, London, UK

${ }^{2}$ Institute of Clinical Sciences, Faculty of Medicine, Imperial College London, London, UK

${ }^{3}$ UCL Cancer Institute, Paul O'Gorman Building, University College London, London, UK

${ }^{4}$ Wellcome Centre for Human Genetics, University of Oxford, Oxford, UK

${ }^{5}$ Institute of Cancer and Genomic Sciences, University of Birmingham, Edgbaston, Birmingham, UK

${ }^{6}$ Cell Cycle Control Team, MRC London Institute of Medical Sciences (LMS), London, UK

* To whom correspondence should be addressed (m.secrier@ucl.ac.uk)

\begin{abstract}
Therapy resistance in cancer is often driven by a subpopulation of cells that are temporarily arrested in a non-proliferative, quiescent or 'dormant' state, which is difficult to capture and whose mutational drivers remain largely unknown. We developed methodology to uniquely identify this state from transcriptomic signals and characterised its prevalence and genomic constraints in solid primary tumours. We show dormancy preferentially emerges in the context of more stable, less mutated genomes which maintain TP53 integrity and lack the hallmarks of DNA damage repair deficiency, while presenting increased APOBEC mutagenesis. We uncover novel genomic dependencies of this process, including the amplification of the centrosomal gene CEP89 as a driver of dormancy impairment. Lastly, we demonstrate that dormancy underlies unfavourable responses to various therapies exploiting cell cycle, kinase signalling and epigenetic mechanisms in single cell data, and propose a signature of dormancy-linked therapeutic resistance to further study and clinically track this state.
\end{abstract}




\section{INTRODUCTION}

2 Quiescence is a widely conserved state of reversible proliferative arrest, essential for the normal

3 development and homeostasis of eukaryotic organisms ${ }^{1,2}$. During quiescence, cells are halted in the

4 G0 phase of the cell cycle, where they maintain minimal basal activity ${ }^{2}$. In cancer, this temporary

5 cell cycle arrest, also termed cellular dormancy, has been proposed as one of the contributing

6 factors of resistance to treatments that target actively dividing cells, such as chemotherapy ${ }^{3-5}$.

7 Moreover, a variety of other pre-existing or acquired resistance scenarios have been explained by a

8 drug-tolerant 'persister' cell state represented by slow cycling or entirely quiescent cells ${ }^{6,7}$. Their

9 presence is associated with minimal residual disease, a major cause of relapse in the clinic ${ }^{8}$. As the

10 neoplastic cells evolve, dormancy can also be employed as a mechanism to facilitate immune

11 evasion ${ }^{9,10}$ or adaptation to new environmental niches during metastatic seeding ${ }^{11,12}$.

12 Despite increasing evidence for the importance of dormancy in cancer progression, it remains a

13 poorly defined biological programme. Hampering our understanding is the fact that quiescence

14 represents a number of heterogeneous states ${ }^{1}$. Canonically, cells can be forced into quiescence

15 through serum starvation, mitogen withdrawal or contact inhibition ${ }^{1}$. Cells can also enter dormancy

16 spontaneously in response to cell-intrinsic factors like replication stress ${ }^{13-15}$. This process is

17 controlled by $\mathrm{p} 53^{16}$, which triggers the inhibition of cyclin-CDK complexes by activating $\mathrm{p} 21^{14}$.

18 This in turn allows the assembly of the DREAM complex - a key effector responsible for

19 repression of cell-cycle dependent gene expression ${ }^{17}$. Min and Spencer ${ }^{18}$ recently demonstrated a

20 much broader systemic coordination of 198 genes underlying distinct types of quiescence by

21 profiling the transcriptomes of cells that entered this state either spontaneously or upon different

22 stimuli. Additionally, proliferation-quiescence decisions can be impacted by oncogenic changes

23 such as $M Y C$ amplification ${ }^{19}$ or altered p38/ERK signalling ${ }^{20}$.

24 Despite these advances, the identification of dormant cells within tumours presents an ongoing 
and maintenance of this state. As they are often defined by a lack of proliferative markers ${ }^{21,22}$,

quiescent cells can be mistaken for other therapy resistant cell types such as senescent or cancer stem cells ${ }^{6,23}$. Unlike quiescent cells, which can readily resume their proliferative state, senescent cells are irreversibly arrested ${ }^{18}$ while cancer stem cells have a high capacity for self-renewal and sit at the top of the differentiation hierarchy ${ }^{24}$. Biomarkers of quiescence that are sufficiently specific and robust to be clinically useful are clearly needed.

32 Furthermore, the extent to which dormancy is enacted through transcriptional or genetic control is

33 unknown ${ }^{5,25}$, as the mutational processes and genomic changes driving quiescence have not been systematically profiled across cancer tissues. Understanding the evolutionary triggers and molecular mechanisms that enable cancer cells to enter and maintain dormancy would enable us to develop pharmacological strategies to selectively eradicate quiescent cancer cells or prevent them from re-entering proliferative cycles.

To address these challenges, we have developed a new method to uniquely and reliably quantify tumour dormancy using transcriptomic data, and employed it to characterise this phenomenon in bulk and single cell datasets from a variety of solid tumours. We identify and validate mutational constraints for dormancy emergence, hinting at potential new therapeutic targets that could exploit this mechanism. We also demonstrate the relevance of dormancy to responses to a range of compounds targeting cell cycle, kinase signalling and epigenetic mechanisms in single cell datasets, and propose an expression signature that could be employed to detect tumour dormancyinduced treatment resistance.

\section{RESULTS}

\section{Evaluating tumour dormancy from transcriptomic data}

49 We hypothesised that primary tumours contain varying numbers of quiescent cells, and this in turn 
51 phenotype, we developed a computational framework that would allow us to quantify dormancy

52 signals in bulk and single cell sequenced cancer samples (Figure 1a). We employed 139

53 differentially expressed genes across five distinct forms of quiescence resulting from serum

54 starvation, contact inhibition, MEK inhibition, CDK4/6 inhibition and spontaneous quiescence, as

55 described by Min and Spencer ${ }^{18}$, as markers of a generic quiescence state. The expression levels of

56 these markers were used to derive a score reflecting the relative level of dormancy within

57 individual tumours (see Methods).

58 We validated multiple scoring methodologies ${ }^{26-29}$ using seven single-cell and bulk datasets ${ }^{30-36}$

59 where actively proliferating and quiescent cells had been independently profiled (Extended Data

60 Figure 1a, Supplementary Table 1, Methods). A combined Z-score approach had the highest

61 accuracy in detecting signals of dormancy, with a 91\% mean performance in classifying cells as

62 quiescent or cycling (Figure 1b, Extended Data Figure 1b). It also outperformed classical cell cycle

63 and dormancy markers, such as the expression of targets of the DREAM complex, CDK2, Ki67

64 and of mini-chromosome replication maintenance (MCM) protein complex genes - which are

65 involved in the initiation of eukaryotic genome replication (Figure 1c). Importantly, our approach

66 provided a good separation between quiescent and proliferating samples across a variety of cancer

67 types and models including cancer cell lines, 3D organoid cultures, circulating tumour cells and

68 patient-derived xenografts (Supplementary Table 1), thereby demonstrating its broad applicability.

69 Furthermore, the strength of the score appeared to reflect the quiescence depth, i.e. the time the

70 cells stay in quiescence ${ }^{37}$ (Figure 1d).

71 We experimentally validated our methodology in nine lung adenocarcinoma cell lines. There was a

72 remarkably good correlation between our predicted quiescence levels and the fraction of quiescent

73 cells, estimated using quantitative, single-cell imaging of phospho-Ser807/811-Rb (PRb, which

74 labels proliferative cells ${ }^{38}$ ) and 24 hour EdU proliferation assays (Figure 1e-h). Cells that were

75 negative for either PRb or EdU were defined as quiescent (see Methods, Figure 1e-f). Importantly, 
a G1 signature (Methods) was not associated with these experimental measurements, proving our method indeed captures quiescence rather than a prolonged G1 state (Figure $1 \mathrm{~g}-\mathrm{h}$ ). The quiescence correlations appeared robust to random removal of individual genes from the signature, with no single gene having an inordinate impact on the score (Extended Data Figure 1c-e). This provided further reassurance that our Z-score based methodology is successful in capturing dormancy signals from bulk tumour data.

82 Having established a robust framework for quantifying tumour dormancy, we next profiled 8,005

83 primary tumour samples across 31 solid cancer tissues from The Cancer Genome Atlas (TCGA).

84 After accounting for potential confounding signals from the microenvironment by correcting for tumour purity (see Methods, Extended Data Figure 1f-g), we observed an entire spectrum of dormancy across tumours that ranged from highly dormant to highly proliferative (Figure 2a).

87 Cancers well known to be frequently dormant, such as glioblastoma ${ }^{3,30}$, were amongst the highest ranked in terms of quiescence, along with kidney and adrenocortical carcinomas (Figure 2b).

89 As expected, tissues with lower stem cell division rates presented a greater propensity for quiescence (Figure 2c) ${ }^{39}$. Importantly, we confirmed that our quiescence scores capture a cellular state that is distinct from that of cancer stem cells, marked by signatures associated with high

92 telomerase activity and an undifferentiated state ${ }^{40,41}$, and that of senescent cells, marked by the

93 Senescence-Associated Secretory Phenotype (SASP) and $\beta$-galactosidase activity ${ }^{42-44}$ (Figure 2d,

94 Extended Data Figures 2a-d). Our scores also showed strong negative correlations with the expression of proliferation markers (Figure 2d).

Lastly, we confirmed dependencies on the p53/p21/DREAM activation axis: tumours that were proficient in TP53 or the components of the DREAM complex, as well as those with higher p21 expression, had elevated dormancy levels across numerous tissues (Figure 2e, Extended Data

99 Figures $2 \mathrm{e}-\mathrm{f}$ ), although only 8 out of 139 dormancy genes are directly transcriptionally regulated by $\mathrm{p} 53^{45}$. Nevertheless, $\mathrm{p} 53$ proficiency appears to be a non-obligatory dependency of dormancy, 
101

102

103

104

105

106

107

108

109

110

111

112

113

114

115

116

117

which is also observed to arise in p53 mutant scenarios in $21 \%$ of cases. p53 has also been shown to play a role in preventing the occurrence of larger structural events and polyploidy ${ }^{46-48}$, potentially explaining the lower dormancy levels we observed in tumours that had undergone whole genome duplication (Figure 2f).

\section{The genomic background of cancer dormancy}

After obtaining dormancy estimates for primary tumour samples, we set out to identify potential genomic triggers which might create a favourable background for tumour dormancy. We identified 285 cancer driver genes that were preferentially altered (via mutations or copy number alterations) either in dormant or fast proliferating tumours (Figure 3a). Reassuringly, this list included genes previously implicated in driving cellular quiescence-proliferation decisions such as TP53 and $M Y C^{16,19}$. An additional 10 cancer genes showed signals of positive selection in the context of high dormancy/proliferation (Supplementary Table 2). We also investigated associations with mutagenic footprints of carcinogens (termed "mutational signatures"), which can be identified as trinucleotide substitution patterns in the genome ${ }^{49,50} .15$ mutational signatures were linked with dormancy either within individual cancer studies or pan-cancer (Extended Data Figure 2g).

Following the initial prioritisation of putative genomic constraints of dormancy, we sought to identify those events that could best distinguish dormant from fast proliferating tumours, while accounting for tissue effects. An ensemble elastic net selection approach similar to the one described by Pich et al ${ }^{51}$ was applied for this purpose (Figure 3b, Methods). Our pan-cancer model identified tissue type to be a major determinant of quiescence levels (Extended Data Figure 3a). It also uncovered a reduced set of 60 genomic events linked with tumour dormancy levels, including SNVs and copy number losses in 17 cancer genes, as well as amplifications of 13 cancer genes (Figure 3c). These events could then be successfully employed to predict quiescence in a separate test dataset, thus internally validating our model (Extended Data Figure 3b). 
Reassuringly, a functional TP53, lack of MYC amplification and lower mutation rates (Figure 3c)

were amongst the top ranked characteristics of dormant tumours, which also displayed less samples. p16 (CDKN2A) deletions, one of the frequent early events during cancer evolution ${ }^{52,53}$, were enriched in dormant tumours. $R B 1$ deletions and amplifications were both associated with a reduction in tumour dormancy, which might reflect the dual role of RB1 in regulating proliferation and apoptosis ${ }^{54}$. Overall, the genomic dependencies of dormancy mainly comprised genes involved in cell cycle pathways, p53 regulation and ubiquitination (most likely of cell cycle targets), TGF-b signalling mediators and RUNX2/3 regulation which have previously been shown to play a role in controlling proliferation and cell cycle entry ${ }^{55}$ (Extended Data Figure 3c).

Our model also calls attention to the broader mutational processes associated with this cellular state. Such processes showed fairly weak and heterogeneous correlations with dormancy within individual cancer tissues (Extended Data Figure 2g), but their contribution becomes substantially clearer pan-cancer once other genomic sources are accounted for. In particular, we identified an association between dormancy and mutagenesis induced by the AID/APOBEC family of cytosine deaminases as denoted by signature $\mathrm{SBS}^{49}$ (Figure 3c). As highlighted by Mas-Ponte and Supek ${ }^{56}$,

143 APOBEC/AID driven mutations tend to be directed towards early-replicating, gene-rich regions of

144 the genome, inducing deleterious events on several genes including ZMYM2, which our pan-cancer model has linked with higher dormancy.

146 In turn, defective DNA mismatch repair, as evidenced by signatures SBS44, SBS20, SBS15,

147 SBS14 and $\mathrm{SBS6}^{49}$, was prevalent in fast cycling tumours (Figure 3c). Mismatch repair

148 deficiencies lead to hypermutation in a phenomenon termed "microsatellite instability" (MSI), which has been linked with increased immune evasion ${ }^{57}$. Cancers particularly prone to MSI include colon, stomach and endometrial carcinomas ${ }^{58}$, where this state was indeed linked with lower 
quiescence (Figure 3d). Furthermore, dormant tumours were depleted of alterations across all DNA damage repair pathways (Figure 3e).

153 Our measurements of dormancy also reflected expected cycling patterns across 27 MCF7 strains ${ }^{59}$ : depletion of PTEN mutations, a dependency highlighted by the pan-cancer model.

156 When checking for dependencies in individual cancer tissues, 27 out of the 28 genes identified by

157 the model were significantly associated with dormancy in at least one tissue, most prominently in breast, lung and liver cancers which also represent the largest studies within TCGA (Figure $3 g$, top panel). Most of these genomic insults were linked with a decrease in dormancy. In external MYC, CEP89 or STAG1, featured most prominently in the largest cohort of breast cancer samples

162 (Figure 3g, bottom panel).

\section{CEP89 amplification contributes to high tumour proliferation rates}

164 To gain more insight into the underlying biology of cellular dormancy, we sought to experimentally validate associations highlighted by the pan-cancer model. We focused on the impact of CEP89 activity on quiescence-proliferation decisions due to the high ranking of this putative oncogene in the model, the relatively unexplored links between CEP89 and cell cycle control, as well as its association with quiescence across a variety of cancer cell lines (Extended Data Figure 4a-c). The function of CEP89 is not well characterised, however, the encoded protein

170 has been proposed to function as a centrosomal-associated protein ${ }^{60,61}$. Centrosomes function as major microtubule-organising centres in cells, playing a key role in mitotic spindle assembly ${ }^{62}$ and

172 the mitotic entry checkpoint ${ }^{63}$. Moreover, centrosomes act as sites of ubiquitin-mediated

173 proteolysis of cell cycle targets ${ }^{64}$, and members of several growth signalling pathways, such as Wnt 
and NF-kB, localise at these structures ${ }^{65,66}$. Several genetic interactions have also been reported between CEP89 and key cell cycle proteins, including cyclin D267 (Figure 4a).

176 Our model linked CEP89 amplification with fast cycling tumours (Figure 3c). Centrosome

177 amplification is a common feature of tumours with high proliferation rates and high genomic

178 instability ${ }^{68}$, and overexpression of centrosomal proteins can alter centriole structure ${ }^{69,70}$. Indeed,

179 CEP89 amplified tumours presented elevated expression of a previously reported centrosome amplification signature (CA20) ${ }^{68}$ (Figure 4b), which was strongly anticorrelated with quiescence levels (Figure 4c). Furthermore, CEP89 expression was prognostic across multiple cancer tissues

Data Figure 4d).

We validated this target in the lung adenocarcinoma cell line NCI-H1299 showing high levels of CEP89 amplification. Cep89 depletion via siRNA knockdown caused a consistent increase in the fraction of quiescent cells as measured by PRb and EdU assays (Figure 4e-f). Thus, we propose CEP89 as a novel cell proliferation regulator that may be exploited in certain scenarios to control tumour growth.

\section{Dormancy in breast cancer}

Due to the variation of dormancy levels across tissues and to increase power, we have taken a pancancer approach to capture genomic features that are universally associated with dormancy.

192 However, we acknowledge that the extremely diverse genomic background for cancer development

193 in different tissues might mean that certain genetic alterations may lead to higher or lower propensity of dormancy in a single tissue only. To investigate this further we focused on breast cancer, the largest cancer dataset available from TCGA, where we still have adequate power and balance of dormant/cycling phenotypes to uncover tissue-specific genomic markers of dormancy. 
could distinguish dormant from fast cycling tumours with a mean accuracy of $74 \%$ (Figure 5a).

199 This model recovered the associations with the APOBEC mutational signature SBS2, TP53

cancer model (Figure 5b). All additional associations validated in the METABRIC cohort and were

also seen in several other cancers, particularly bladder, lung and lower grade glioma (Figure 5c).

203 Since APOBEC/AID activity has been linked with dormancy in both the pan-cancer and the tissue-

specific models, we further investigated its role in breast cancer, where the associated mutation

signature SBS2 is relatively prevalent. Indeed, breast cancer samples enriched in APOBEC-

higher quiescence levels (Figure 5d). Across breast cancer subtypes, the Luminal A subtype showed the highest levels of dormancy, however, Her2+ tumours had the highest incidence of

APOBEC associated mutational events, suggesting this subtype may be the most susceptible to

APOBEC-linked quiescence (Figure 5e-f).

\section{Characterisation of individual quiescence programmes}

212 While we had previously examined a generic dormancy programme, cancer cells can enter

213 quiescence due to different stimuli ${ }^{1}$ and this may inform its aetiology and manifestation. To

214 explore this, we re-scored tumours based on gene expression programmes specific to serum

215 starvation, contact inhibition, MEK inhibition, CDK4/6 inhibition or spontaneously occurring

216 quiescence as defined by Min and Spencer ${ }^{18}$ (see Methods). We observed a good correlation

217 between the dormancy estimates representing individual quiescence programmes and the

218 expression of genes associated with the corresponding form of quiescence in the literature (Figure

219 6a-e, Extended Data Figure 5). CDK4/6 inhibition-induced quiescence levels were further

220 validated using external RNA-seq datasets from cancer cell lines and xenograft mice sequenced

221 before and after treatment with the CDK4/6 inhibitor Palbociclib ${ }^{71,72}$ (Figure 6f, Supplementary 
222 Table 4). Interestingly, we also observed significant differences in spontaneous quiescence scores

223 before and after treatment. Indeed, p21 activity has been linked with the Palbociclib mechanism of

224 action $^{73,74}$, and this analysis suggests potential similarities between CDK4/6 inhibition and p21-

225 dependent quiescence phenotypes.

226 Having validated our framework for quantifying stimulus-specific quiescence programmes, we

227 proceeded to estimate the dominant form of quiescence in different cancer types (Figure $6 \mathrm{~g}$ ). We

228 found a range of quiescence aetiologies across most tissues, while a minority of cancers were

229 dominated by a single form of quiescence, e.g. serum starvation in all dormant

230 pheochromocytomas and paragangliomas, contact inhibition in $88 \%$ of head and neck carcinomas

231 and CDK4/6 inhibition in $80 \%$ of dormant adrenocortical carcinomas. While we do not wish to

232 claim that the dormancy state will have necessarily been induced by the actual predicted stimulus

233 (impossible in the case of CDK4/6 or MEK inhibition, as the analysed samples are all treatment-

234 naïve), we suggest that the downstream signalling cascade may resemble that triggered by such

235 stimuli, e.g. via CDK4/6 or MEK loss of function mutations. Amongst these states, spontaneous

236 quiescence appeared most strongly dependent on p53 functionality (Supplementary Table 3). This

237 points to common transcriptional features of drug-tolerant dormant cells in different cancer settings

238 that could be employed in designing ways to eradicate these cells in the future.

\section{Clinical relevance of tumour dormancy}

240 Overall, dormancy appears to be beneficial for the long-term outcome of cancer patients (Figure

241 7a, Extended Data Figure 6a). As expected, quiescence levels were increased in stage 1 tumours,

242 although later stages also exhibited occasional elevated dormancy (Extended Data Figure 6b).

243 Indeed, such dormant, indolent tumours could have higher chances of being eradicated earlier in

244 the disease, which is consistent with reported worse prognosis of patients with higher tumour cell

245 proliferation rates and less stable, more mutated genomes ${ }^{75}$. However, outcomes do vary depending 
on the quiescence subtype, with worse survival observed upon contact inhibition (Figure 7b). The

249 While dormancy may confer an overall survival advantage in most cancers, it can also provide a pool of cells that are capable of developing resistance to therapy ${ }^{34,76}$. Using our methodology, we indeed observed an increase in quiescence levels in cell lines following treatment with EGFR, BRAF and CDK4/6 inhibitors, as well as conventionally used chemotherapies such as 5-

Fluorouracil (5-FU) in multiple bulk RNA-seq datasets (Figure 7d). Furthermore, the recent

Using our quiescence signature and single-cell data from RKO and SW480 colon cancer cell lines chemotherapy treatment. Within the p53 proficient cell line RKO, the fraction of quiescent cells increased from $41 \%$ to $93 \%$ after treatment with a low dose $(10 \mu \mathrm{M})$ of 5-FU and persisted at

SW480 cells, further emphasizing the key role of p53 as a regulator of quiescence (Figure $7 \mathrm{~g}-\mathrm{h}$ ).

SW480 cells showed higher apoptotic activity following treatment compared to RKO cells,

265 Similarly, using single cell data from an EGFR mutant Non-Small Cell Lung Cancer (NSCLC) cell line treated with the EGFR inhibitor Erlotinib ${ }^{78}$, we predicted that $40 \%$ of cells were likely to exist

267 in a dormant state prior to treatment. EGFR inhibition led to a massive decrease in cell numbers 268 immediately after treatment, mostly due to proliferating cells dying off (Extended Data Figures 6e- 
proliferating again in the following days during continuous treatment, with the percentage of proliferating cells approaching pre-treatment levels by day 11 (Figure 7j). The same trend captured by our signature could be observed upon KRAS and BRAF inhibition in different cell line models (Extended Data Figure 6g-j, Supplementary Table 4) ${ }^{34,78}$. Furthermore, during the first days of treatment the NSCLC cells that survived EGFR inhibition appeared to reside in a state most resembling that induced by serum starvation (Figures 7k-1). Both EGFR kinase inhibitors and serum starvation have been shown to trigger autophagy ${ }^{79}$, which may explain the convergence between this inhibitory trigger and the type of quiescence response. At day 11 most of the remaining dormant cells appeared in a state similar to that preceding the treatment (Figure 71).

Thus, quiescence appears to explain resistance to broad acting chemotherapy agents as well as targeted molecular inhibitors of the Ras/MAPK signalling pathway, being either selected for, or induced immediately upon treatment, and gradually waning over time as cells start re-entering the cell cycle. Using massively multiplexed chemical transcriptomic data, we also analysed responses to 188 small molecule inhibitors in cell lines at single-cell resolution ${ }^{80}$ (Extended Data Figure 7). We observed a large increase in quiescence following treatment with compounds targeting cell cycle regulation and tyrosine kinase signalling, consistent with our previous results, but also for compounds modulating epigenetic regulation, e.g. histone deacetylase inhibitors - thus highlighting the broad relevance of quiescence.

These analyses showcase our ability to probe the nature of quiescent phenotypes using a defined dormancy signature, which could aid the development of methods to selectively target quiescent drug-resistant persister cells. However, a major challenge of scRNA-seq data analysis is the high percentage of gene dropout, which could impact our ability to evaluate dormancy using the full 139 gene signature. The scRNA-seq datasets we analysed exhibited an average drop-out of 8.5 genes out of the full gene signature. While our scoring method remains robust to such levels of dropout (Extended Data Figure 1c-e), we also employed machine learning to reduce our initial list of 139 
markers of dormancy to a robust 35-gene signature, comprised mainly of RNA metabolism and

splicing-regulating factors, but also of genes involved in cell cycle progression, ageing and

senescence, which could be applied to sparser datasets with larger levels of gene dropout (Methods,

Figure 8a-b). The optimised signature of dormancy performed similarly to the initial broadly

defined programme in distinguishing highly dormant and fast cycling tumours (Figure 8c), showed scenarios.

\section{DISCUSSION}

Despite its crucial role in cancer progression and resistance to therapies, dormancy has remained poorly characterised due to the scarcity of suitable models and biomarkers for large-scale tracking in the tissue or blood. Lack of proliferative markers such as Ki67 or CDK2 21,81 does not uniquely

311 distinguish quiescence from other cell cycle phases, e.g. G1 or S. This study overcame this

312 limitation by employing genes active in different forms of quiescence whose patterns of expression are distinct from markers of a longer G1 phase, senescence or stemness. We demonstrate a robust method for profiling this signature to identify dormancy in bulk and single cell RNA-seq data, its versatility evidenced by high classification accuracies across a variety of solid cancer datasets. More variable performance was observed when applied to hematopoietic stem cells as it was not designed to capture signals in this context (Extended Data Figure 1b).

318 We show that dormancy is pervasive across different solid cancers and generally associated with more stable, less mutated genomes with intact DNA damage repair pathways. We also find a link 
321 affecting a variety of genes such as PTEN, CEP89, CYLD, LMNA that appear unfavourable to

322 dormancy development, thus potentially implicating them in influencing quiescence-proliferation

323 decisions. Among these, we propose CEP89 amplification as novel biomarker of non-small cell

324 lung primary tumours with low quiescence capacity. Genomic alterations enriched in dormant

325 tumours, such as p16 or ZMYM2 deletions, were more rare and predominantly captured pan-cancer.

326 Such targets should be further validated and could be exploited to either counteract dormancy or

327 induce it.

328 Our finding that primary tumour dormancy confers a favourable prognosis could raise a potential

329 limitation of our approach in distinguishing between slow-dividing and drug-tolerant persister cell

330 phenotypes $^{6}$ in bulk transcriptome data. Nevertheless, our methodology is directly extendable to

331 single cell data, where we demonstrate a clear association between existent/emerging quiescence

332 and resistance to multiple cell cycle, kinase signalling and epigenetic targeting regimens in a

333 variety of cancers. Quiescent cells utilise several mechanisms to achieve drug resistance, including

334 upregulation of stress-induced pathways such as anti-apoptotic BCL-2 signalling ${ }^{82}$, anti-ROS

335 programmes $^{18}$ or immune evasion ${ }^{10}$. Further studies are needed to elucidate if these mechanisms

336 are specific to individual cancer tissues or individual quiescence types.

337 Overall, our study provides for the first time a pan-cancer view of dormancy and its evolutionary

338 constraints, underlying novel mutational dependencies which could be exploited in the clinic. We

339 propose a tumour dormancy signature which can be measured in bulk tissue or single cells, and

340 could inform therapeutic strategies or risk of relapse. This signature could be assessed in the clinic

341 to track rapidly emerging resistance, e.g. through liquid biopsies or targeted gene panels. We hope

342 these insights can be used as building blocks for future studies into the different regulators of

343 dormancy, including epigenetics and microenvironmental interactions, as well as the mechanisms

344 by which it enables therapeutic resistance both in solid and haematological malignancies. 


\section{ONLINE METHODS}

\section{Selection of quiescence marker genes}

348 Generic quiescence markers:

349 Differential expression analysis results comparing cycling immortalised, non-transformed human

350 epithelial cells and cells in five different forms of quiescence (spontaneous quiescence, contact

351 inhibition, serum starvation, CDK4/6 inhibition and MEK inhibition) were obtained from Min and

352 Spencer ${ }^{18}$. A total of 195 genes were differentially expressed in all five forms of quiescence under 353 an adjusted p-value cut-off of 0.05 . This gene list, reflective of a generic quiescence phenotype,

354 was subjected to the following refinement and filtering steps: (1) selection of genes with a

355 unidirectional change of expression across all five forms of quiescence; (2) removal of genes

356 involved in other cell cycle stages included in the "KEGG_CELL_CYCLE" gene list deposited at

357 MSigDB; (3) removal of genes showing low standard deviation and low levels of expression within

358 the TCGA dataset, or which showed low correlation with the pan-cancer expression of the

359 transcriptional targets of the DREAM complex, the main effector of quiescence, in TCGA.

360 Quiescence subtype-specific markers:

361 Gene lists representing spontaneous quiescence, contact inhibition, serum starvation, CDK4/6

362 inhibition and MEK inhibition programmes were obtained using genes differentially expressed in

363 each individual quiescence form using an adjusted p-value cutoff of 0.05 . The gene lists were

364 subjected to filtering steps 2 and 3 described above. Following the refinement steps, 10 upregulated

365 and 10 downregulated genes with highest $\log 2$ fold changes were selected for each quiescence

366 type. 


\section{Dormancy quantification}

369 The GSVA R package was used to implement the combined z-score ${ }^{26}$, ssGSEA ${ }^{27}$ and GSVA ${ }^{28}$ gene set enrichment methods. For the above three methods a separate score was obtained for genes upregulated in quiescence and genes downregulated in quiescence, following which a final quiescence score was obtained by subtracting the two scores. The singscore single-sample gene signature scoring method ${ }^{29}$ was implemented using the singscore $\mathrm{R}$ package. In addition to these, we also calculated a mean scaled quiescence score based on the refined list of genes upregulated and downregulated in quiescence, as well as a curated housekeeping genes from the “HSIAO_HOUSEKEEPING_GENES” list deposited at MSigDB, as follows:

$$
\mathrm{QS}=\frac{\frac{1}{\mathrm{n}} \sum G_{U}-\frac{1}{\mathrm{n}} \sum G_{D}}{\frac{1}{\mathrm{n}} \sum G_{H}}
$$

378 QS = mean scale quiescence score

$379 \mathrm{G}_{\mathrm{U}}=$ expression of genes upregulated in quiescence

$380 \mathrm{G}_{\mathrm{D}}=$ expression of genes downregulated in quiescence

$381 \mathrm{G}_{\mathrm{H}}=$ expression of housekeeping genes

$382 \mathrm{n}=$ number of genes in each gene set

383 Quiescence scores for the TCGA cohort were derived from expression data scaled by tumour purity

384 estimates. The pan-cancer TCGA samples were also classified into "high" or "low" quiescence

385 groups based on $\mathrm{k}-$ means clustering $(\mathrm{k}=2)$ on the expression data of 139 quiescence biomarker

386 genes, following the removal of tissue-specific expression differences using the ComBat function

387 from the $s v a$ R package ${ }^{83}$. 
389 We employed the GSE124109 dataset from Fujimaki et al ${ }^{37}$ where rat embryonic fibroblasts were 390 transcriptomically profiled as they moved from shallow to deep quiescence over time in the

391 absence of growth signals. The derived quiescence scores using our combined z-score

392 methodology increased from shallow to deep quiescence.

\section{Validation of quiescence scoring methodologies}

394 Single-cell RNA-sequencing validation datasets:

395 Datasets were obtained from the ArrayExpress and Gene Expression Omnibus (GEO) databases 396 though the following GEO Series accession numbers: GSE83142, GSE75367, GSE137912,

397 GSE139013, GSE90742 and E-MTAB-4547. Quality control analysis was standardised using the SingleCellExperiment ${ }^{84}$ and scater $^{85} \mathrm{R}$ packages. Normalisation was performed using the scran $^{86} \mathrm{R}$ 399 package.

Bulk RNA-sequencing validation datasets:

401 Datasets were obtained from the GEO database through the following GEO Series accession 402 numbers: GSE93391, GSE114012, GSE131594, GSE152699, GSE124854, GSE135215, 403 GSE99116 and GSE124109. GSE114012 count data were normalised to TPM values using the 404 GeoTcgaData R package. All normalised datasets were log-transformed before further analysis.

405 The accuracy with which the quiescence scoring methods could separate proliferating and 406 quiescent samples within the validation datasets was determined by calculating the area under the

407 curve of the receiver operating characteristic (ROC) curves, using the plotROC R package. 


\section{Experimental validation in lung adenocarcinoma cell lines}

410 The average fraction of cancer cells spontaneously entering quiescence was estimated for nine lung

411 adenocarcinoma cell lines (NCIH460, A549, NCIH1666, NCIH1944, NCIH1563, NCIH1299,

412 NCIH1650, H358, L23) using EdU and phospho-Rb staining proliferation assays.

413 Cell lines were obtained from ATCC or Sigma and regularly checked for mycoplasma. A549 and

414 NCIH460 were cultured in DMEM (Gibco). NCIH358, NCIH1299 and NCIH1563 were

415 maintained in RPMI-1640 (Gibco) supplemented with 5mM sodium pyruvate and $0.5 \%$ glucose.

416 NCIH1944, NCIH1666, NCIH1650 and L23 were grown in RPMI-1640 ATCC formulation

417 (Gibco). A427 were cultured in EMEM (ATCC). A549, NCIH460, H358, NCIH1299, NCIH1563,

418 A427 were supplemented with 10\% heat inactivated FBS. NCIH1666 with 5\% heat-inactivated

419 FBS and all other cell lines with 10\% non-heat inactivated FBS. All cell lines had penicillin-

420 streptomycin (Gibco) added to $1 \%$. Cells were maintained at $37^{\circ} \mathrm{C}$ and $5 \% \mathrm{CO}_{2}$. To calculate the quiescent fraction, A549 and NCIH460 cells were plated at a density of 500 cells/well, and all other cell lines at a density of 1000/well, in 384well CellCarrier Ultra plates (PerkinElmer) in the relevant media. $24 \mathrm{~h}$ later, $5 \mu \mathrm{M}$ EdU was added and cells were incubated for a further $24 \mathrm{~h}$ before

424 fixing in a final concentration of $4 \%$ formaldehyde (15 min, RT), permeabilization with PBS/ $0.5 \%$

425 Triton X-100 (15 min, RT) and blocking with 2\% BSA in PBS (60 min, RT). The EdU signal was 426 detected using Click-iT chemistry, according to the manufacturer's protocol (ThermoFisher). Cells 427 were also labelled for phospho-Ser807/811 Rb (PRb) using Rabbit mAb 8516 (CST) at 1:2000 in 428 blocking solution, overnight at $4^{\circ} \mathrm{C}$. Unbound primary antibody was washed three times in PBS 429 and secondary Alexa-conjugated antibodies were used to detect the signal (ThermoFisher, 1:1000, $4301 \mathrm{~h}$ at RT). Finally nuclei were labelled with Hoechst $33258(1 \mu \mathrm{g} / \mathrm{ml}, 15 \mathrm{~min}$ RT $)$ before imaging 431 on a high-content widefield Operetta microscope, 20x N.A. 0.8. Automated image analysis 432 (Harmony, PerkinElmer) was used to segment and quantify nuclear signals in imaged cells. 
433 Quiescent cells were defined by the absence of EdU or PRb staining, determined by quantification

434 of their nuclear expression (Figure 1e-f).

435 The quiescence scores for cancer cell lines were calculated using corresponding log-transformed

436 RPKM normalised bulk RNA-seq data from the Cancer Cell Line Encyclopedia (CCLE)

437 database ${ }^{87}$.

438 CEP89 was depleted by ON-Target siRNA Pool from Horizon. NCI-H1299 cells were reverse

439 transfected in 384 well plates with $20 \mathrm{nM}$ of Non-targeting control (NTC) or CEP89-targeting

440 siRNA using Lipofectamine RNAiMax (ThermoFisher), according to the manufacturer's

441 instructions. Cells were left for $24 \mathrm{~h}$, before $5 \mu \mathrm{M}$ EdU was added for the final $24 \mathrm{~h}$ and then cells

442 were processed as above to determine the quiescent fraction. To determine the level of Cep89

443 depletion by western blot, cells were reverse transfected with siRNA in 24 well plates. $48 \mathrm{~h}$ after

444 transfection, cells were lysed directly in 1x SDS sample buffer with 1mM DTT (ThermoFisher).

445 Samples were separated on pre-cast 4-20\% Tris-Glycine gels, transferred to PVDF using the iBlot2 446 system and membranes blocked in blocking buffer (5\% milk in TBS) for $1 \mathrm{~h}$ at RT. The membrane

447 was then cut and the upper half was incubated in 1:1000 Cep89 antibody (Sigma, HPA040056), the

448 bottom half in B-actin antibody 1:2000 (CST; 3700S) diluted in blocking buffer overnight at 4'C.

449 Membranes were washed three times in TBS- $0.05 \%$ TritonX-100 before being incubated in

450 secondary anti-rabbit (Cep89) or anti-mouse (B-actin) HRP conjugated antibodies (CST 7074P2

451 and CST 7076P2, respectively) diluted 1:2000 in blocking buffer for $1 \mathrm{~h}$ at RT. Membranes were

452 washed three times again and signal detected using Clarity ECL solution (BioRad) and scanned on

453 an Amersham ImageQuant 800 analyser.

\section{Multi-omics discovery cohort}

455 FPKM normalised RNA-sequencing expression data, copy number variation gene-level data, as

456 well as mutation annotation files aligned against the GRCh38 human reference genome from the 
457 Mutect2 pipeline were downloaded using the TCGABiolinks $\mathrm{R}$ package ${ }^{88}$ for 9,712 TCGA primary

458 tumour samples across 31 solid cancer types. Haematological malignancies were excluded as the

459 quiescence markers were derived in epithelial cells and might not be equally suited to capture this

460 phenotype in blood. For patients with multiple samples available, one RNA-seq barcode entry was

461 selected for each individual patient resulting in 9,631 total entries. All expression data were log-

462 transformed for downstream analysis. During quiescence score calculation, expression data for the

463 primary tumour samples was scaled according to tumour purity estimates reported by Hoadley et

$464 \mathrm{al}^{89}$ to account for potential confounding quiescence signals coming from non-tumour cells in the

465 microenvironment. Samples with purity estimates lower than 30\% were removed, leaving 8,005

466 samples for downstream analysis.

467 The mutation rates of all TCGA primary tumour samples were determined by log-transforming the

468 total number of mutations in each sample divided by the length of the exome capture (38Mb).

469 TP53 functional status was assessed based on somatic mutation and copy number alterations as

470 described in Zhang et $\mathrm{al}^{90}$. TP53 mutation and copy number for the TCGA tumours were

471 downloaded from cBioPortal (http://www.cbioportal.org). Tumours with TP53 oncogenic

472 mutations (annotated by OncoKB) and copy-number alterations (GISTIC score $\leq-1$ ) were assigned

473 as TP53 mutant and CNV loss. Tumours without these TP53 alterations were assigned as TP53

474 wild type. The effects of the TP53 mutation status on quiescence score were then determined with

475 a linear model approach with the quiescence score as a dependent variable and mutational status as

476 an independent variable. The $P$ values were FDR-adjusted.

477 APOBEC mutagenesis enriched samples were determined through pan-cancer clustering of

478 mutational signature contributions as described in Wiecek et $\mathrm{al}^{91}$. The APOBEC mutagenesis

479 cluster was defined as the cluster with highest mean SBS2 and SBS13 contribution. This was

480 repeated 100 times and only samples which appeared in the APOBEC cluster at least 50 times were 481 counted as being APOBEC enriched. 
482 Aneuploidy scores and whole genome duplication events across TCGA samples were obtained

483 from Taylor et $\mathrm{al}^{92}$. Microsatellite instability status for uterine corpus endometrial carcinoma, as

484 well as stomach and colon adenocarcinoma samples were obtained from Cortes-Ciriano et $\mathrm{al}^{58}$.

485 Telomerase enzymatic activity "EXTEND” scores were obtained from Noureen et al ${ }^{40}$. Expression-

486 based cancer cell stemness indices were obtained from Malta et al ${ }^{41}$. Centrosome amplification

487 transcriptomic signature (CA20) scores were obtained from Almeida et al ${ }^{68}$.

488 PHATE dimensionality reduction

489 The phateR $\mathrm{R}$ package ${ }^{93}$ was used to perform the dimensionality reduction with a constant seed for 490 reproducibility. The ComBat function from the sva $\mathrm{R}$ package ${ }^{94}$ was used to remove tissue-specific 491 expression patterns from the TCGA RNA-seq data.

\section{Cancer stem cell division estimates}

493 The mean stem cell division estimates for different cancer types used in this study were obtained

494 from Tomasetti and Vogelstein ${ }^{39}$.

\section{Positive selection analysis}

496 Genes positively selected specifically in samples clustered into low or high dormancy groups were

497 identified based on $\mathrm{dN} / \mathrm{dS}$ analysis using the $d N d S c v$ R package ${ }^{95}$, run with default parameters.

498 Genes showing signals of positive selection in either the high dormancy or low dormancy samples

499 which encoded olfactory receptors were discarded from downstream analysis.

\section{Mutational signature estimation}

501 Mutational signature contributions were inferred as described in Wiecek et al ${ }^{91}$. 


\section{Ensemble elastic net regression model}

504 The COSMIC database was used to source a list of 723 known drivers of tumorigenesis (Tiers

$5051+2) .285$ oncogenes and tumour suppressors from a curated list showed a significant enrichment

506 or depletion of mutations or copy number variants in dormant samples either pan-cancer or within

507 individual TCGA studies.

508 To classify dormant from fast proliferating tumours, the 286 genes were used as input features for

509 an ensemble elastic net regression model along the tumour mutational rate, whole-genome

510 doubling estimates, ploidy, aneuploidy scores, 10 positively selected genes and 15 mutational

511 signatures, which showed a significant correlation with quiescence scores either pan-cancer or

512 within individual TCGA studies. The caret R package was used to build an elastic net regression

513 model 1000 times on the training dataset of 3,753 TCGA primary tumour samples ( $80 \%$ of the total

514 dataset). Only samples with at least 50 mutations were used in the model, for which mutational

515 signatures could be reliably estimated. For each of the 1000 iterations, we randomly selected $90 \%$

516 of the samples from the training dataset to build the model. Only features which were included in

517 all 1000 model iterations were selected for further analysis. To test the performance of our

518 approach, a linear regression model was built using the reduced list of genomic features and their

519 corresponding coefficients averaged across the 1000 elastic net regression model iterations. When

520 applying the resulting linear regression model on the internal validation dataset of 936 samples, we

521 found a strong correlation between the observed and predicted quiescence scores $(\mathrm{R}=0.73, \mathrm{p}<$

$522 \quad 2.2 \mathrm{e}-16)$.

523 SHAP values for the linear regression model used to predict quiescence scores were obtained using

524 the fastshap R package. 


\section{Gene enrichment and network analysis}

527 Gene set enrichment analysis was carried out using the ReactomePA R package, as well as

528 GeneMania $^{96}$ and ConsensusPathDB ${ }^{97}$. Interactions between CEP89 and other cell cycle

529 components were inferred using the list of cell cycle genes provided by cBioPortal and GeneMania

530 to reconstruct the expanded network with direct interactors (STAG1, CCND2, STAT3). Networks

531 were visualised using Cytoscape ${ }^{98}$.

\section{Gene lists}

533 Genes associated with the G1 phase of the cell cycle were obtained from the curated

534 “REACTOME_G1_PHASE “ list deposited at MSigDB.

535 Genes associated with apoptosis were obtained from the curated "HALLMARK_APOPTOSIS" list 536 deposited at MSigDB.

537 Genes associated with the senescence-associated secretory phenotype were obtained from Basisty

538 et $\mathrm{al}^{44}$. Lists of genes making up the various DNA damage repair pathways were derived from

539 Pearl et al ${ }^{99}$.

540 Genes associated with contact inhibition were obtained from the curated "contact inhibition" gene

541 ontology term. Genes associated with serum starvation were obtained from the curated

542 “REACTOME_CELLULAR_RESPONSE_TO_STARVATION” list deposited at MSigDB. MEK

543 inhibition was assessed based on the activity of the MAPK pathway as determined using an

544 expression signature (MPAS) consisting of 10 downstream MAPK transcripts ${ }^{100}$.

\section{Validation of genomic features of dormancy}

546 For elastic net model feature validation, RNA-seq data was downloaded for six cancer studies from

547 cBioPortal ${ }^{101}$, along with patient-matched whole-genome, whole-exome and targeted sequencing 
548 data. The 6 datasets used comprise breast cancer (SMC ${ }^{102}$ and METABRIC ${ }^{103}$ ), paediatric Wilms'

549 tumor (TARGET ${ }^{104}$ ), bladder cancer, prostate adenocarcinoma and sarcoma (MSKCC $\left.{ }^{105-107}\right)$

550 studies. The data were processed and analysed in the same manner as the TCGA data. RNA-seq

551 data for 27 MCF7 cell line strains, alongside cell line growth rates and targeted mutational

552 sequencing data were obtained from Ben-David et al ${ }^{59}$.

\section{Genomic dependency modelling in breast cancer}

554 An ANOVA-based feature importance classification was used and identified 30 genomic features

555 most discriminative of samples with lower and higher than average quiescence scores. A random

556 forest model was then built using the identified features and correctly classified samples according

557 to their quiescence state with a mean accuracy of $74 \%$ across five randomly sampled test datasets

558 from the cohort.

\section{Survival analysis}

560 Multivariate Cox Proportional Hazards analysis was carried out using the coxph function from the 561 survival $\mathrm{R}$ package. The optimal quiescence score cut-off value of 2.95 was determined using the 562 surv_cutpoint function.

\section{Treatment response scRNA-seq and bulk RNA-seq data}

564 Datasets have been obtained from the GEO database through the following GEO Series accession

565 numbers: GSE134836, GSE134838, GSE134839, GSE137912, GSE149224, GSE124854,

566 GSE135215, GSE99116, GSE152699, GSE178839 and GSE139944. The umap R package was

567 used for dimensionality reduction with constant seed for reproducibility. 


\section{Quiescence subtype determination}

570 TCGA cohort studies:

571 Samples with evidence of dormancy characterised by a generic quiescence score $>0$ were further

572 subclassified based on the most likely form of quiescence exhibited, among CDK4/6 inhibition,

573 contact inhibition, MEK inhibition, spontaneous quiescence or serum starvation, using subtype-

574 specific expression signatures. We opted for a conservative approach and classed each dormant

575 sample into a specific quiescence subtype if the quiescence score for the corresponding programme

576 was higher than one standard deviation of the distribution across the TCGA cohort, and if the score

577 was significantly higher than for the remaining programmes when assessed using a Student's t test.

578 Samples which could not be classified into any of the five quiescence states characterised in this

579 study were classified as "uncertain".

580 Single-cell RNA seq treatment response datasets:

581 The quiescence subtype of individual single cells was inferred by mapping such individual cells

582 onto the reference dataset of MCF10A cells reflecting different forms of quiescence obtained from

583 Min and Spencer ${ }^{18}$. The ComBat R package was used to remove the study batch effect between the 584 expression data to be classified and the reference bulk RNA-seq data. PCA dimensionality

585 reduction analysis was then used on the combined datasets using the prcomp $\mathrm{R}$ function. For each

586 patient sample or single-cell expression data entry, a k-nearest neighbour algorithm classification

587 was performed using the knn function from the class R package. During the classification the three

588 nearest reference bulk RNA-seq data points were considered, with two nearest neighbours with

589 identical class needed for classification. 
593 We investigated if a subset of the 139 quiescence-related genes could act as a more reliable marker

594 of dormancy that would bypass dropout issues in single cell data. This was performed in three 595 steps:

We collected three high confidence single cell expression datasets separating quiescent from quiescence state of a given cell based on the expression levels of the 139 genes. The Gini indices corresponding to each gene in the model were normalised to a range of values between state relative to the other 138 genes. The procedure was repeated 1000 times for each of the three datasets, and the average Gini coefficients across iterations were stored.

(2) Prioritisation of gene subsets based on cumulative importance in the model different gene combinations were produced. genes in the 10 lung cancer cell lines previously employed for experimental validation, along with the quiescence state of the lines as inferred by PRb and EdU staining. For each gene subset, a combined z-score of quiescence was calculated from the expression levels as described previously. The correlations between this z-score and the two experimental measurements of quiescence were used to establish the ability of a gene combination to predict 
quiescence. Among the top performing subsets, a 35 gene signature with a mean correlation of $78 \%$ between predicted and measured quiescence levels in the test data $(p=0.016)$ showed the highest correlation with PRb measurements capturing short-lived quiescence, the more common state observed in single cell treatment datasets. Therefore, this signature was deemed to achieve the best trade-off between gene numbers and signal capture.

621 The optimised gene signature is available at https://github.com/secrierlab/CancerDormancy

\section{Data visualization and basic statistics}

623 Graphs were generated using the ggplot2 and ggpubr R packages. Groups were compared using a

624 two-sided Student's t test, Wilcoxon rank-sum test or ANOVA, as appropriate. P-values were

625 adjusted for multiple testing where appropriate using the Benjamini-Hochberg method.

\section{Data availability}

627 The results published here are in part based upon data generated by the TCGA Research Network:

628 https://www.cancer.gov/tcga, METABRIC (https://ega-archive.org/studies/EGAS00000000083),

629 MSK-IMPACT (https://www.mskcc.org/msk-impact), or deposited at cBioPortal

630 (https://www.cbioportal.org/) and GEO (https://www.ncbi.nlm.nih.gov/geo/). All data comply with 631 ethical regulations, with approval and informed consent for collection and sharing already obtained 632 by the relevant consortia.

\section{Code availability}

634 All code developed for the purpose of this study can be found at the following repository: 


\section{FUNDING}

638 AJW and DHJ were supported by MRC DTP grants (MR/N013867/1). MPC was supported by an

639 Academy of Medical Science Springboard award (SBF004\1042). GMT was supported by a

640 Wellcome Seed Award in Science (215296/Z/19/Z). MS was supported by a UKRI Future Leaders

641 Fellowship (MR/T042184/1). Work in MS's lab was supported by a BBSRC equipment grant

642 (BB/R01356X/1) and a Wellcome Institutional Strategic Support Fund (204841/Z/16/Z). ARB and

643 SC are supported by a CRUK CDF (C63833/A25729) and work in ARB's lab is supported by

644 MRC core-funding to the London Institute of Medical Sciences (MC-A658-5TY60).

\section{ACKNOWLEDGEMENTS}

646 We would like to thank Prof Chris Barnes for the very helpful discussions and input on the findings 647 of the study.

\section{AUTHOR CONTRIBUTIONS}

649 MS designed the study and supervised the computational analyses. ARB designed and supervised

650 the experimental validation in cell lines. GB supervised the analysis of p53 functional association.

651 AJW developed the dormancy scoring methodology and performed all computational analyses to

652 validate and apply it in bulk and single cell datasets, as well as link it to genomic features. SC

653 performed the experimental validation of quiescence prevalence and CEP89 association with

654 quiescence in cell lines. DK performed the inference of the minimal signature of dormancy

655 applicable in single cell data. MPC performed the random forest modelling and feature selection in

656 breast cancer. LG performed the positive selection analysis. GMT wrote the code for batch effect correction and for PCA mapping of single cell data on a reference dataset. DHJ performed the

658 APOBEC enrichment classification. PZ and LX performed the quiescence comparison of p53 wild

659 type and mutated cancers. MS, AJW, ARB and SC wrote the manuscript, with contributions from

660 all other authors. All authors read and approved the manuscript. 


\section{COMPETING INTEREST STATEMENT}

662

None declared.

\section{3}

664

665

666

667

668

669

670

671

672

673

674

675

676

677

678

679

680

681

682

683

684

685

686

687

688

689

690

691

692

693

694

695

696

697

698

699

700

701

702

703

704

705

706

707

708

709

\section{REFERENCES}

1. Coller, H.A., Sang, L. \& Roberts, J.M. A new description of cellular quiescence. PLoS Biol 4, e83 (2006).

2. van Dijk, D. et al. Slow-growing cells within isogenic populations have increased RNA polymerase error rates and DNA damage. Nat Commun 6, 7972 (2015).

3. Chen, J. et al. A restricted cell population propagates glioblastoma growth after chemotherapy. Nature 488, 522-6 (2012).

4. Puig, I. et al. TET2 controls chemoresistant slow-cycling cancer cell survival and tumor recurrence. J Clin Invest 128, 3887-3905 (2018).

5. Rehman, S.K. et al. Colorectal Cancer Cells Enter a Diapause-like DTP State to Survive Chemotherapy. Cell 184, 226-242.e21 (2021).

6. Phan, T.G. \& Croucher, P.I. The dormant cancer cell life cycle. Nat Rev Cancer 20, 398-411 (2020).

7. Cabanos, H.F. \& Hata, A.N. Emerging Insights into Targeted Therapy-Tolerant Persister Cells in Cancer. Cancers (Basel) 13(2021).

8. Masago, K., Fujita, S. \& Yatabe, Y. Targeting minimal residual disease after surgery with molecular targeted therapy: the real path to a cure? J Thorac Dis 10, S1982-S1985 (2018).

9. Malladi, S. et al. Metastatic Latency and Immune Evasion through Autocrine Inhibition of WNT. Cell 165, 45-60 (2016).

10. Ribas, A. Adaptive Immune Resistance: How Cancer Protects from Immune Attack. Cancer Discov 5, 915-9 (2015).

11. Sosa, M.S., Bragado, P. \& Aguirre-Ghiso, J.A. Mechanisms of disseminated cancer cell dormancy: an awakening field. Nat Rev Cancer 14, 611-22 (2014).

12. Barkan, D. et al. Metastatic growth from dormant cells induced by a col-I-enriched fibrotic environment. Cancer Res 70, 5706-16 (2010).

13. Arora, M., Moser, J., Phadke, H., Basha, A.A. \& Spencer, S.L. Endogenous Replication Stress in Mother Cells Leads to Quiescence of Daughter Cells. Cell Rep 19, 1351-1364 (2017).

14. Barr, A.R. et al. DNA damage during S-phase mediates the proliferation-quiescence decision in the subsequent G1 via p21 expression. Nat Commun 8, 14728 (2017).

15. Heldt, F.S., Barr, A.R., Cooper, S., Bakal, C. \& Novák, B. A comprehensive model for the proliferation-quiescence decision in response to endogenous DNA damage in human cells. Proc Natl Acad Sci U S A 115, 2532-2537 (2018).

16. Itahana, K. et al. A role for p53 in maintaining and establishing the quiescence growth arrest in human cells. J Biol Chem 277, 18206-14 (2002).

17. Sadasivam, S. \& DeCaprio, J.A. The DREAM complex: master coordinator of cell cycle-dependent gene expression. Nature Reviews Cancer 13, 585-595 (2013).

18. Min, M. \& Spencer, S.L. Spontaneously slow-cycling subpopulations of human cells originate from activation of stress-response pathways. PLoS Biol 17, e3000178 (2019).

19. García-Gutiérrez, L., Delgado, M.D. \& León, J. MYC Oncogene Contributions to Release of Cell Cycle Brakes. Genes (Basel) 10(2019).

20. Aguirre-Ghiso, J.A., Estrada, Y., Liu, D. \& Ossowski, L. ERK(MAPK) activity as a determinant of tumor growth and dormancy; regulation by p38(SAPK). Cancer Res 63, 1684-95 (2003).

21. Spencer, S.L. et al. The proliferation-quiescence decision is controlled by a bifurcation in CDK2 activity at mitotic exit. Cell 155, 369-83 (2013).

22. Miller, I. et al. Ki67 is a Graded Rather than a Binary Marker of Proliferation versus Quiescence. Cell Rep 24, 1105-1112.e5 (2018).

23. Reya, T., Morrison, S.J., Clarke, M.F. \& Weissman, I.L. Stem cells, cancer, and cancer stem cells. Nature 414, 105-11 (2001). 
710

711

712

713

714

715

716

717

718

719

720

721

722

723

724

725

726

727

728

729

730

731

732

733

734

735

736

737

738

739

740

741

742

743

744

745

746

747

748

749

750

751

752

753

754

755

756

757

758

759

760

761

762

763

764

24. Kleinsmith, L.J. \& Pierce, G.B., Jr. MULTIPOTENTIALITY OF SINGLE EMBRYONAL CARCINOMA CELLS. Cancer Res 24, 1544-51 (1964).

25. Turati, V.A. et al. Chemotherapy induces canalization of cell state in childhood B-cell precursor acute lymphoblastic leukemia. Nature Cancer (2021).

26. Lee, E., Chuang, H.Y., Kim, J.W., Ideker, T. \& Lee, D. Inferring pathway activity toward precise disease classification. PLoS Comput Biol 4, e1000217 (2008).

27. Barbie, D.A. et al. Systematic RNA interference reveals that oncogenic KRAS-driven cancers require TBK1. Nature 462, 108-12 (2009).

28. Hänzelmann, S., Castelo, R. \& Guinney, J. GSVA: gene set variation analysis for microarray and RNA-seq data. BMC Bioinformatics 14, 7 (2013).

29. Foroutan, M. et al. Single sample scoring of molecular phenotypes. BMC Bioinformatics 19, 404 (2018).

30. Aulestia, F.J. et al. Quiescence status of glioblastoma stem-like cells involves remodelling of $\mathrm{Ca}(2+)$ signalling and mitochondrial shape. Sci Rep 8, 9731 (2018).

31. Kurppa, K.J. et al. Treatment-Induced Tumor Dormancy through YAP-Mediated Transcriptional Reprogramming of the Apoptotic Pathway. Cancer Cell 37, 104-122.e12 (2020).

32. Ebinger, S. et al. Characterization of Rare, Dormant, and Therapy-Resistant Cells in Acute Lymphoblastic Leukemia. Cancer Cell 30, 849-862 (2016).

33. Jordan, N.V. et al. HER2 expression identifies dynamic functional states within circulating breast cancer cells. Nature 537, 102-106 (2016).

34. Xue, J.Y. et al. Rapid non-uniform adaptation to conformation-specific KRAS(G12C) inhibition. Nature 577, 421-425 (2020).

35. Buczacki, S.J.A. et al. Itraconazole targets cell cycle heterogeneity in colorectal cancer. $J$ Exp Med 215, 1891-1912 (2018).

36. Grigore, F. et al. BRAF inhibition in melanoma is associated with the dysregulation of histone methylation and histone methyltransferases. Neoplasia 22, 376-389 (2020).

37. Fujimaki, K. et al. Graded regulation of cellular quiescence depth between proliferation and senescence by a lysosomal dimmer switch. Proc Natl Acad Sci U S A 116, 22624-22634 (2019).

38. Stallaert, W. et al. The structure of the human cell cycle. bioRxiv, 2021.02.11.430845 (2021).

39. Tomasetti, C. \& Vogelstein, B. Cancer etiology. Variation in cancer risk among tissues can be explained by the number of stem cell divisions. Science 347, 78-81 (2015).

40. Noureen, N. et al. Integrated analysis of telomerase enzymatic activity unravels an association with cancer stemness and proliferation. Nature Communications 12, 139 (2021).

41. Malta, T.M. et al. Machine Learning Identifies Stemness Features Associated with Oncogenic Dedifferentiation. Cell 173, 338-354.e15 (2018).

42. Dimri, G.P. et al. A biomarker that identifies senescent human cells in culture and in aging skin in vivo. Proceedings of the National Academy of Sciences 92, 9363 (1995).

43. Debacq-Chainiaux, F., Erusalimsky, J.D., Campisi, J. \& Toussaint, O. Protocols to detect senescence-associated beta-galactosidase (SA-betagal) activity, a biomarker of senescent cells in culture and in vivo. Nat Protoc 4, 1798-806 (2009).

44. Basisty, N. et al. A proteomic atlas of senescence-associated secretomes for aging biomarker development. PLOS Biology 18, e3000599 (2020).

45. Fischer, M. Census and evaluation of p53 target genes. Oncogene 36, 3943-3956 (2017).

46. Andreassen, P.R., Lohez, O.D., Lacroix, F.B. \& Margolis, R.L. Tetraploid state induces p53dependent arrest of nontransformed mammalian cells in G1. Molecular biology of the cell 12, 13151328 (2001).

47. Di Leonardo, A. et al. DNA rereplication in the presence of mitotic spindle inhibitors in human and mouse fibroblasts lacking either p53 or pRb function. Cancer Res 57, 1013-9 (1997).

48. Ganem, N.J. et al. Cytokinesis failure triggers hippo tumor suppressor pathway activation. Cell 158, 833-848 (2014).

49. Alexandrov, L.B. et al. The repertoire of mutational signatures in human cancer. Nature 578, 94101 (2020).

50. Alexandrov, L.B. et al. Signatures of mutational processes in human cancer. Nature 500, 415-421 (2013).

51. Pich, O. et al. The mutational footprints of cancer therapies. Nat Genet 51, 1732-1740 (2019). 
52. Maley, C.C. \& Reid, B.J. Natural selection in neoplastic progression of Barrett's esophagus. Semin Cancer Biol 15, 474-83 (2005).

53. Bardeesy, N. \& DePinho, R.A. Pancreatic cancer biology and genetics. Nat Rev Cancer 2, 897-909 (2002).

54. Indovina, P., Pentimalli, F., Casini, N., Vocca, I. \& Giordano, A. RB1 dual role in proliferation and apoptosis: cell fate control and implications for cancer therapy. Oncotarget 6(2015).

55. Miyazono, K., Maeda, S. \& Imamura, T. Coordinate regulation of cell growth and differentiation by TGF-beta superfamily and Runx proteins. Oncogene 23, $4232-7$ (2004).

56. Mas-Ponte, D. \& Supek, F. DNA mismatch repair promotes APOBEC3-mediated diffuse hypermutation in human cancers. Nat Genet 52, 958-968 (2020).

57. Kloor, M. \& von Knebel Doeberitz, M. The Immune Biology of Microsatellite-Unstable Cancer. Trends Cancer 2, 121-133 (2016).

58. Cortes-Ciriano, I., Lee, S., Park, W.-Y., Kim, T.-M. \& Park, P.J. A molecular portrait of microsatellite instability across multiple cancers. Nature Communications 8, 15180 (2017).

59. Ben-David, U. et al. Genetic and transcriptional evolution alters cancer cell line drug response. Nature 560, 325-330 (2018).

60. Jakobsen, L. et al. Novel asymmetrically localizing components of human centrosomes identified by complementary proteomics methods. Embo j 30, 1520-35 (2011).

61. Sillibourne, J.E. et al. Assessing the localization of centrosomal proteins by PALM/STORM nanoscopy. Cytoskeleton (Hoboken) 68, 619-27 (2011).

62. Bettencourt-Dias, M. \& Glover, D.M. Centrosome biogenesis and function: centrosomics brings new understanding. Nature Reviews Molecular Cell Biology 8, 451-463 (2007).

63. Grallert, A. et al. Removal of centrosomal PP1 by NIMA kinase unlocks the MPF feedback loop to promote mitotic commitment in S. pombe. Curr Biol 23, 213-22 (2013).

64. Wigley, W.C. et al. Dynamic association of proteasomal machinery with the centrosome. The Journal of cell biology 145, 481-490 (1999).

65. Itoh, K., Jenny, A., Mlodzik, M. \& Sokol, S.Y. Centrosomal localization of Diversin and its relevance to Wnt signaling. J Cell Sci 122, 3791-8 (2009).

66. Kfoury, Y. et al. Ubiquitylated Tax targets and binds the IKK signalosome at the centrosome. Oncogene 27, 1665-1676 (2008).

67. Lin, A., Wang, R.T., Ahn, S., Park, C.C. \& Smith, D.J. A genome-wide map of human genetic interactions inferred from radiation hybrid genotypes. Genome Res 20, 1122-32 (2010).

68. de Almeida, B.P., Vieira, A.F., Paredes, J., Bettencourt-Dias, M. \& Barbosa-Morais, N.L. Pancancer association of a centrosome amplification gene expression signature with genomic alterations and clinical outcome. PLOS Computational Biology 15, e1006832 (2019).

69. Kohlmaier, G. et al. Overly long centrioles and defective cell division upon excess of the SAS-4related protein CPAP. Curr Biol 19, 1012-8 (2009).

70. Tang, C.-J.C., Fu, R.-H., Wu, K.-S., Hsu, W.-B. \& Tang, T.K. CPAP is a cell-cycle regulated protein that controls centriole length. Nature Cell Biology 11, 825-831 (2009).

71. Hafner, M. et al. Multiomics Profiling Establishes the Polypharmacology of FDA-Approved CDK4/6 Inhibitors and the Potential for Differential Clinical Activity. Cell Chem Biol 26, 10671080.e8 (2019).

72. Salvador-Barbero, B. et al. CDK4/6 Inhibitors Impair Recovery from Cytotoxic Chemotherapy in Pancreatic Adenocarcinoma. Cancer Cell 37, 340-353.e6 (2020).

73. Guiley, K.Z. et al. p27 allosterically activates cyclin-dependent kinase 4 and antagonizes palbociclib inhibition. Science 366(2019).

74. Pack, L.R., Daigh, L.H., Chung, M. \& Meyer, T. Clinical CDK4/6 inhibitors induce selective and immediate dissociation of $\mathrm{p} 21$ from cyclin D-CDK4 to inhibit CDK2. Nat Commun 12, 3356 (2021).

75. Zhu, X. et al. The prognostic and predictive potential of Ki-67 in triple-negative breast cancer. Scientific Reports 10, 225 (2020).

76. Brown, J.A. et al. TGF- $\beta$-Induced Quiescence Mediates Chemoresistance of Tumor-Propagating Cells in Squamous Cell Carcinoma. Cell Stem Cell 21, 650-664.e8 (2017).

77. $\quad$ Park, S.R. et al. Single-Cell Transcriptome Analysis of Colon Cancer Cell Response to 5Fluorouracil-Induced DNA Damage. Cell Rep 32, 108077 (2020). 
78. Aissa, A.F. et al. Single-cell transcriptional changes associated with drug tolerance and response to

combination therapies in cancer. Nat Commun 12, 1628 (2021).

79. Tan, X., Thapa, N., Sun, Y. \& Anderson, R.A. A kinase-independent role for EGF receptor in autophagy initiation. Cell 160, 145-60 (2015).

80. Srivatsan, S.R. et al. Massively multiplex chemical transcriptomics at single-cell resolution. Science 367, 45-51 (2020).

81. Moser, J., Miller, I., Carter, D. \& Spencer, S.L. Control of the Restriction Point by Rb and p21. Proc Natl Acad Sci U S A 115, E8219-e8227 (2018).

82. Minassian, L.M., Cotechini, T., Huitema, E. \& Graham, C.H. Hypoxia-Induced Resistance to Chemotherapy in Cancer. Adv Exp Med Biol 1136, 123-139 (2019).

83. Johnson, W.E., Li, C. \& Rabinovic, A. Adjusting batch effects in microarray expression data using empirical Bayes methods. Biostatistics 8, 118-27 (2007).

84. Amezquita, R.A. et al. Orchestrating single-cell analysis with Bioconductor. Nature Methods 17, 137-145 (2020).

85. McCarthy, D.J., Campbell, K.R., Lun, A.T.L. \& Wills, Q.F. Scater: pre-processing, quality control, normalization and visualization of single-cell RNA-seq data in R. Bioinformatics 33, 1179-1186 (2017).

86. Lun, A.T., McCarthy, D.J. \& Marioni, J.C. A step-by-step workflow for low-level analysis of single-cell RNA-seq data with Bioconductor. F1000Res 5, 2122 (2016).

87. Barretina, J. et al. The Cancer Cell Line Encyclopedia enables predictive modelling of anticancer drug sensitivity. Nature 483, 603-7 (2012).

88. Colaprico, A. et al. TCGAbiolinks: an R/Bioconductor package for integrative analysis of TCGA data. Nucleic Acids Res 44, e71 (2016).

89. Hoadley, K.A. et al. Cell-of-Origin Patterns Dominate the Molecular Classification of 10,000 Tumors from 33 Types of Cancer. Cell 173, 291-304.e6 (2018).

90. Zhang, P. et al. Germline and Somatic Genetic Variants in the p53 Pathway Interact to Affect Cancer Risk, Progression, and Drug Response. Cancer Res 81, 1667-1680 (2021).

91. Wiecek, A.J., Jacobson, D.H., Lason, W. \& Secrier, M. Pan-Cancer Survey of Tumor Mass Dormancy and Underlying Mutational Processes. Frontiers in Cell and Developmental Biology 9, 1820 (2021).

92. Taylor, A.M. et al. Genomic and Functional Approaches to Understanding Cancer Aneuploidy. Cancer Cell 33, 676-689.e3 (2018).

93. Moon, K.R. et al. Visualizing structure and transitions in high-dimensional biological data. Nature biotechnology 37, 1482-1492 (2019).

94. Leek, J.T., Johnson, W.E., Parker, H.S., Jaffe, A.E. \& Storey, J.D. The sva package for removing batch effects and other unwanted variation in high-throughput experiments. Bioinformatics 28, 8823 (2012).

95. Martincorena, I. et al. Universal Patterns of Selection in Cancer and Somatic Tissues. Cell 171, 1029-1041.e21 (2017).

96. Warde-Farley, D. et al. The GeneMANIA prediction server: biological network integration for gene prioritization and predicting gene function. Nucleic Acids Res 38, W214-20 (2010).

97. Kamburov, A. et al. ConsensusPathDB: toward a more complete picture of cell biology. Nucleic Acids Res 39, D712-7 (2011).

98. Shannon, P. et al. Cytoscape: a software environment for integrated models of biomolecular interaction networks. Genome Res 13, 2498-504 (2003).

99. Pearl, L.H., Schierz, A.C., Ward, S.E., Al-Lazikani, B. \& Pearl, F.M.G. Therapeutic opportunities within the DNA damage response. Nature Reviews Cancer 15, 166-180 (2015).

100. Wagle, M.C. et al. A transcriptional MAPK Pathway Activity Score (MPAS) is a clinically relevant biomarker in multiple cancer types. NPJ Precis Oncol 2, 7 (2018).

101. Cerami, E. et al. The cBio cancer genomics portal: an open platform for exploring multidimensional cancer genomics data. Cancer Discov 2, 401-4 (2012).

102. Kan, Z. et al. Multi-omics profiling of younger Asian breast cancers reveals distinctive molecular signatures. Nat Commun 9, 1725 (2018).

103. Curtis, C. et al. The genomic and transcriptomic architecture of 2,000 breast tumours reveals novel subgroups. Nature 486, 346-52 (2012). 
bioRxiv preprint doi: https://doi.org/10.1101/2021.11.12.468410; this version posted December 3, 2021. The copyright holder for this

preprint (which was not certified by peer review) is the author/funder, who has granted bioRxiv a license to display the preprint in perpetuity. It is made available under aCC-BY-NC-ND 4.0 International license.

104. Gadd, S. et al. A Children's Oncology Group and TARGET initiative exploring the genetic landscape of Wilms tumor. Nat Genet 49, 1487-1494 (2017).

105. Iyer, G. et al. Prevalence and co-occurrence of actionable genomic alterations in high-grade bladder cancer. J Clin Oncol 31, 3133-40 (2013).

106. Taylor, B.S. et al. Integrative genomic profiling of human prostate cancer. Cancer Cell 18, 11-22 (2010).

107. Barretina, J. et al. Subtype-specific genomic alterations define new targets for soft-tissue sarcoma therapy. Nat Genet 42, 715-21 (2010). 
bioRxiv preprint doi: https://doi.org/10.1101/2021.11.12.468410; this version posted December 3, 2021. The copyright holder for this

preprint (which was not certified by peer review) is the author/funder, who has granted bioRxiv a license to display the preprint in perpetuity. It is made available under aCC-BY-NC-ND 4.0 International license.

FIGURES
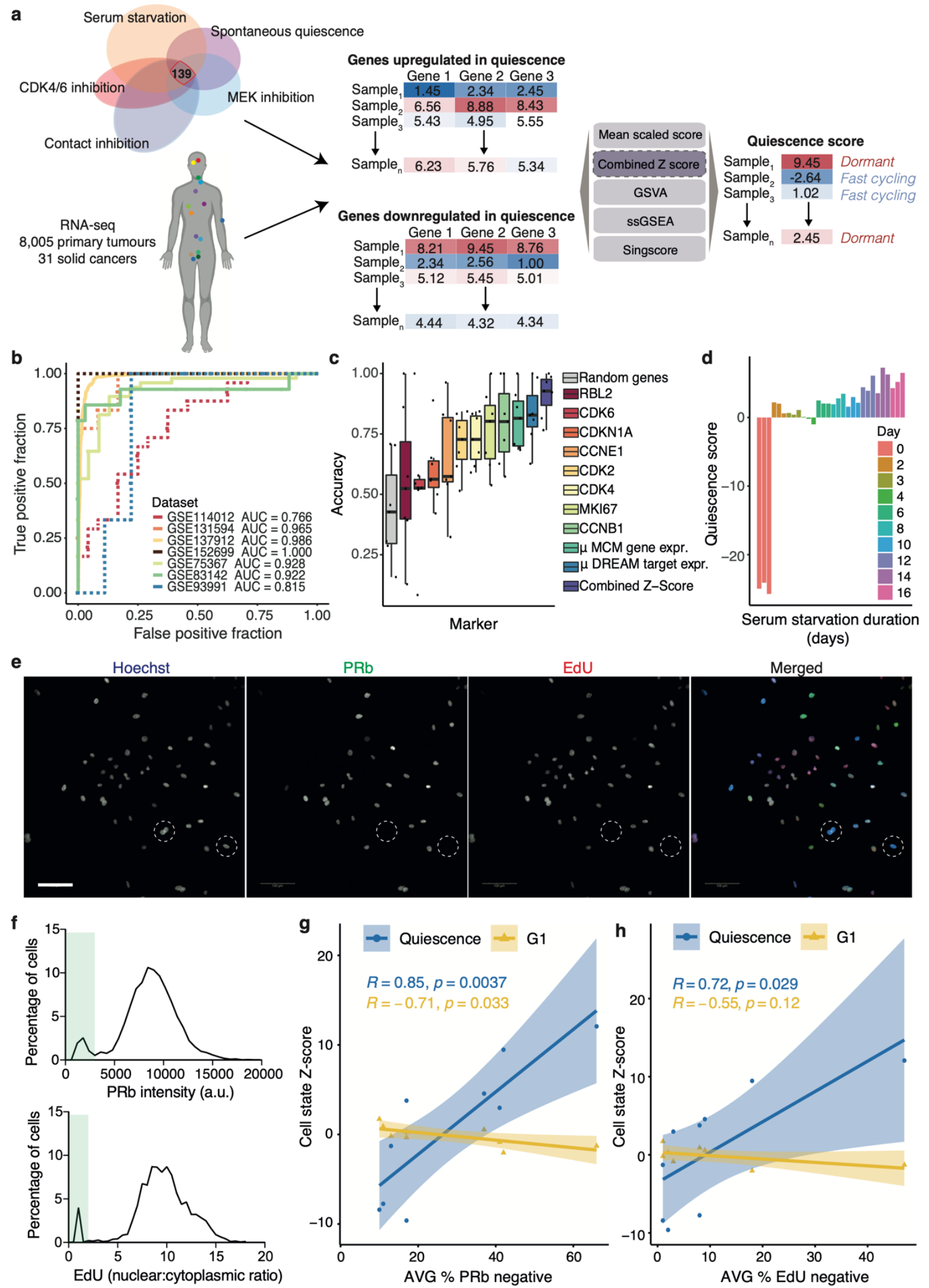

886 Figure 1: Methodology for quantifying cancer dormancy. (a) Workflow for evaluating

887 dormancy from RNA-seq data. 139 genes differentially expressed in multiple forms of quiescence 
were employed to score dormancy across cancer tissues. (b) Receiver operating characteristic

(ROC) curves illustrating the performance of the z-score methodology on separating actively approach and classic cell proliferation markers across the seven single-cell/bulk RNA-seq validation datasets. (d) Quiescence levels of embryonic fibroblast cells under serum starvation for various amounts of time. Replicates are depicted in the same colour. (e) Representative images of

895 lung cancer cell lines immunostained and analysed to detect the quiescent fraction. Hoechst (labels 896 all nuclei) is in blue, $\mathrm{PRb}$ in green and EdU in red in merged image. White dashed circles highlight

897 quiescent cells that are negative for both PRb and EdU signals. Scale bar: $100 \mu \mathrm{m}$. (f) Graphs show 898 single cell quantification of PRb and EdU intensities taken from images and used to define the cut899 off to calculate the quiescent fraction (QF; green boxes). Images in (e) and graphs in (f) are taken

900 from the A549 cell line. (g-h) Correlation between theoretical estimates of a quiescence or G1 state

901 and the fraction of cells entering quiescence in nine lung adenocarcinoma cell lines, as assessed

902 through (g) phospho-Rb assays and (h) EdU assays. Mean of $n=3$ is shown for the average

903 percentage of quiescent cells. 

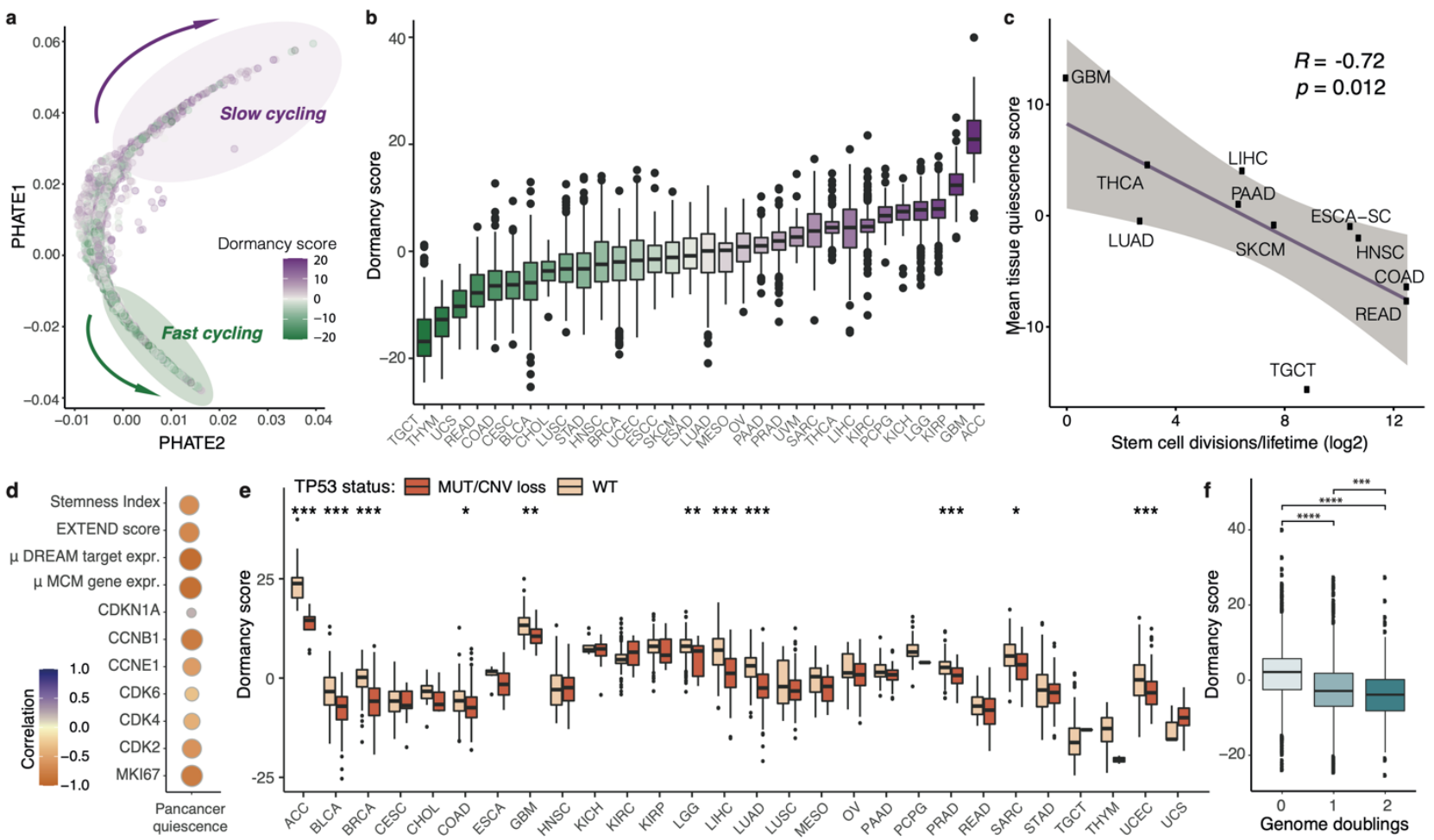

906

Figure 2: Pan-cancer evaluation of dormancy. (a) PHATE plot illustrating the wide spectrum of

907 dormancy-proliferation states across 8,005 primary tumour samples from TCGA. Each sample is

908 coloured according to the relative dormancy level. (b) Variation in tumour dormancy levels across

909 different cancer tissues. (c) Correlation between mean quiescence scores and stem cell division

910 estimates for various tissue types. (d) Correlating tumour dormancy scores with cancer cell

911 stemness (Stemness Index), telomerase activity (EXTEND score) and the expression of several

912 commonly used proliferation markers. The Pearson correlation coefficient is displayed. RC -

913 replication complex. (e) Consistently higher levels of dormancy are detected in samples with

914 functional p53. (f) Lower quiescence scores are observed in tumours with one or two whole

915 genome duplication events. Wilcoxon rank-sum test $\mathrm{p}$-values are displayed in boxplots, ${ }^{*} \mathrm{p}<0.05$;

916

$* *_{\mathrm{p}}<0.01 ; * * *_{\mathrm{p}}<0.001 ; * * * * \mathrm{p}<0.0001$ 
bioRxiv preprint doi: https://doi.org/10.1101/2021.11.12.468410; this version posted December 3, 2021. The copyright holder for this

preprint (which was not certified by peer review) is the author/funder, who has granted bioRxiv a license to display the preprint in perpetuity. It is made available under aCC-BY-NC-ND 4.0 International license.

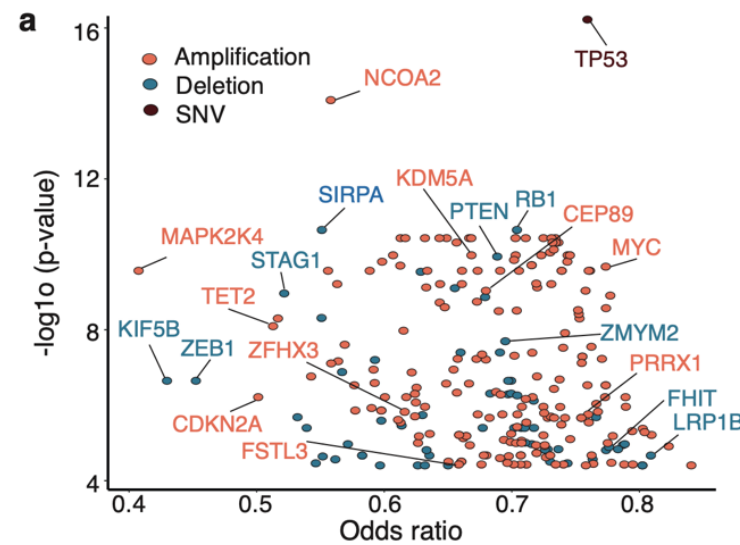

C

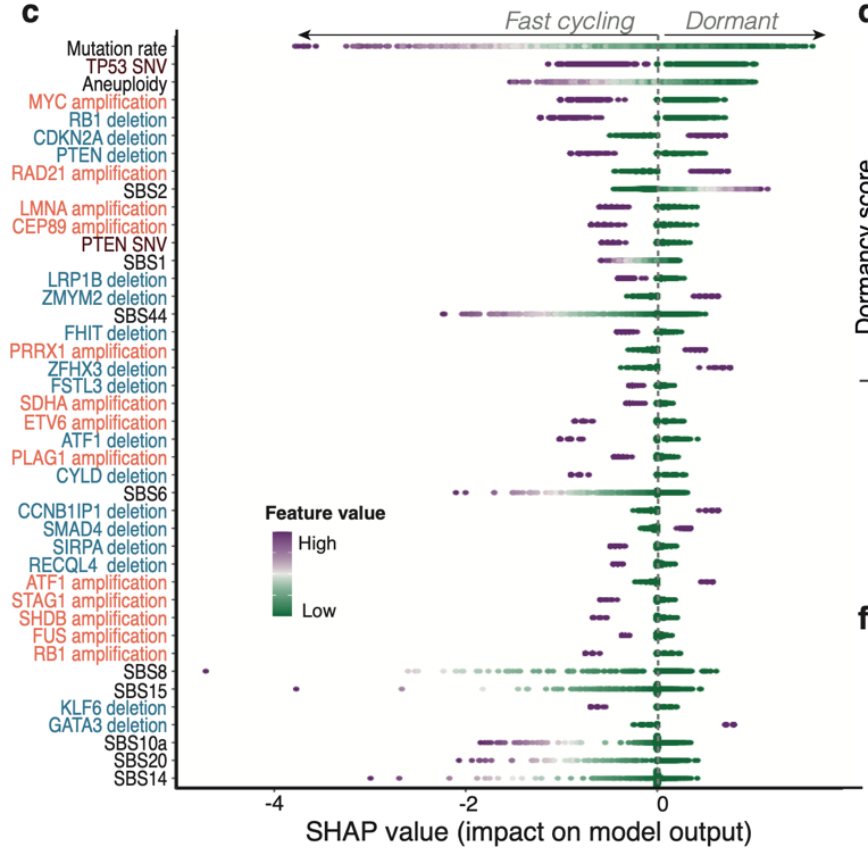

b

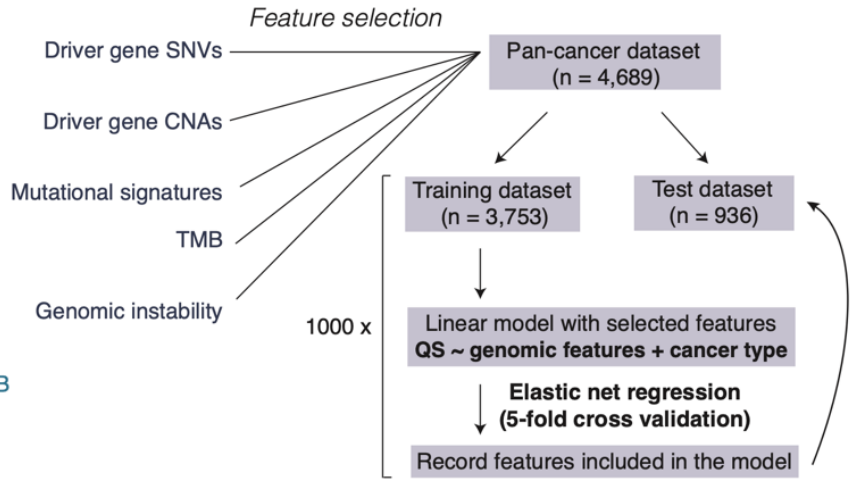

e

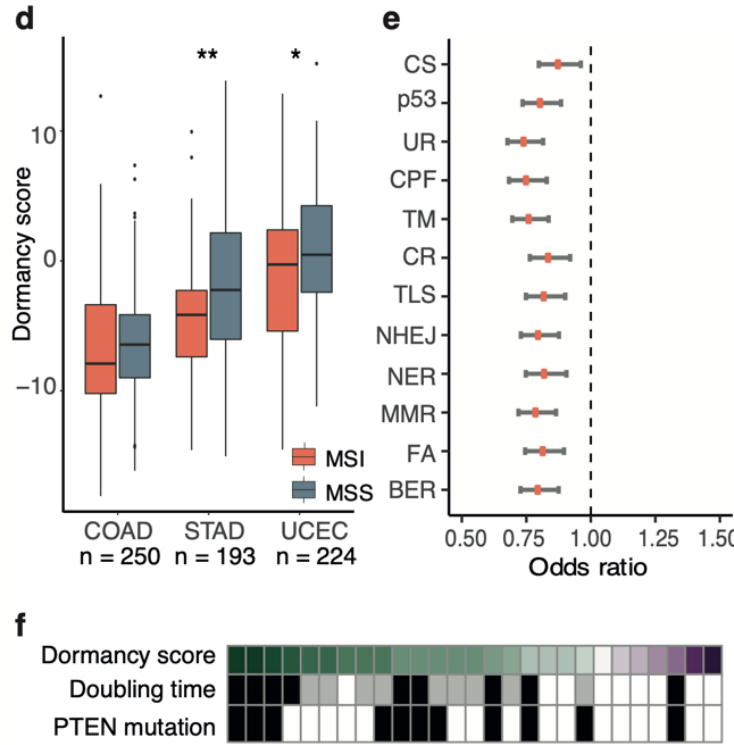

घFast $\square$ Medium $\square$ Slow

g
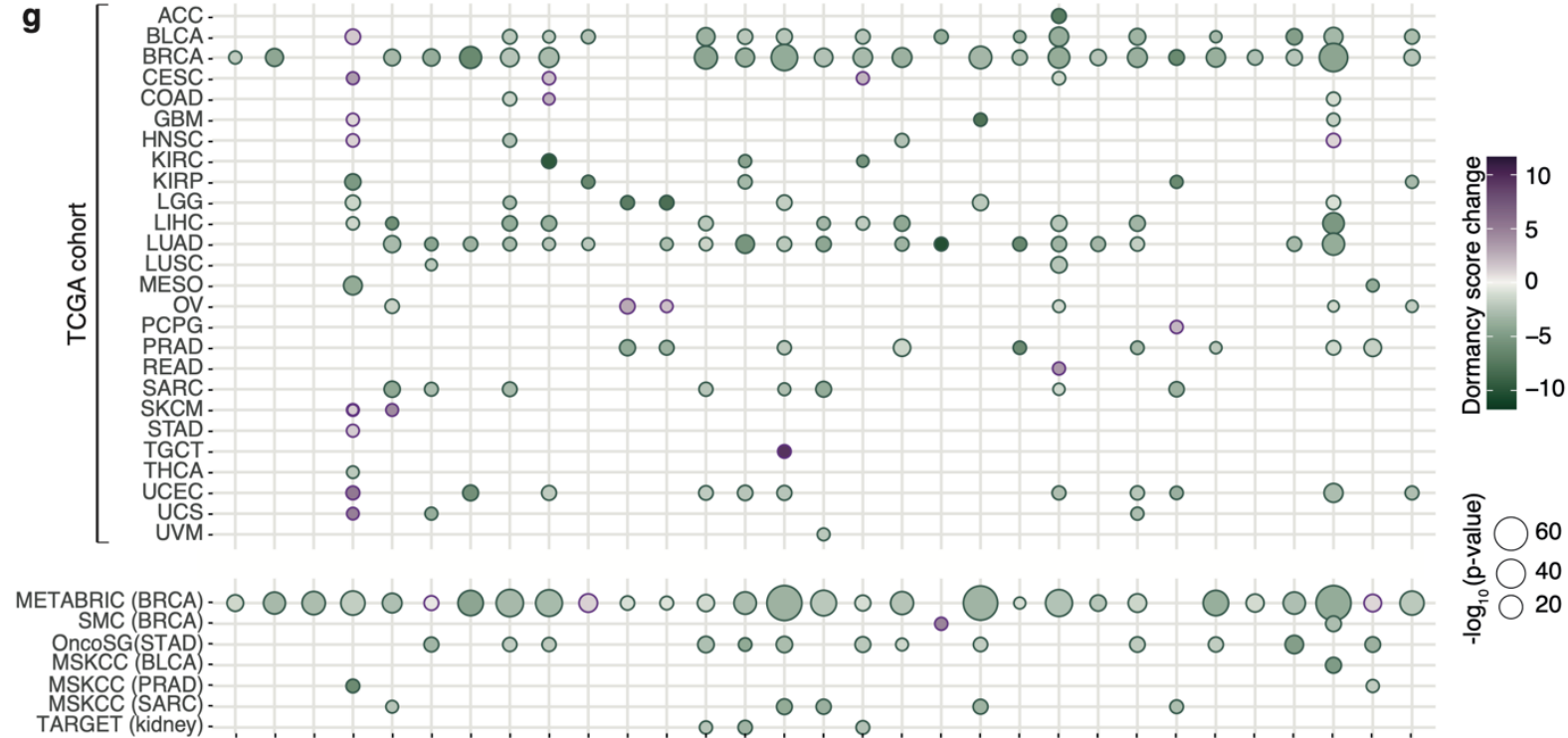

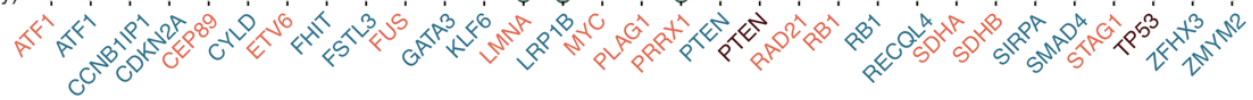

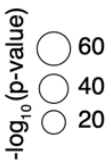

918 Figure 3: Pan-cancer genomic landscape of dormancy. (a) Cancer drivers with mutations or 
the pan-cancer model are highlighted. (b) Schematic of the ensemble elastic net modelling employed to prioritise genomic changes associated with tumour dormancy. (c) Genomic events

922 significantly associated with dormancy, ranked according to their importance in the model (highest

923 to lowest). Each point depicts an individual tumour sample, coloured by the value of the respective

924 feature. For discrete variables purple indicates the presence of the feature and green its absence.

925 The Shapley values indicate the impact of individual feature values on the quiescence score

926 prediction. (d) Dormancy levels are significantly reduced in microsatellite unstable (MSI) samples

927 in stomach adenocarcinoma (STAD) and uterine corpus endometrial carcinoma (UCEC), with the

928 same trend (albeit not significant) shown in colon adenocarcinoma (COAD). Wilcoxon rank-sum

929 test $* \mathrm{p}<0.05 ; * \mathrm{*}<0.01$. (e) Genomic alterations are depleted across DNA repair pathways during

930 dormancy. Odds ratios of mutational load on pathway in dormancy are depicted, along with

931 confidence intervals. $\mathrm{CS}=$ chromosome segregation; $\mathrm{p} 53=\mathrm{p} 53$ pathway; $\mathrm{UR}=$ ubiquitylation

932 response; $\mathrm{CPF}=$ checkpoint factors; $\mathrm{TM}=$ telomere maintenance; $\mathrm{CR}=$ chromatin remodelling;

933 TLS=translesion synthesis; NHEJ=non-homologous end joining; $\mathrm{NER}=$ =nucleotide excision repair;

$934 \mathrm{MMR}=$ mismatch repair; $\mathrm{FA}=$ Fanconi Anaemia; $\mathrm{BER}=$ base excision repair. (f) Dormancy is

935 increased in cell lines with slow doubling time across MCF7 strains, which also show lower

936 prevalence of PTEN mutations. (g) Tissue-specific changes in quiescence between samples

937 with/without dormancy-associated deletions (blue), amplifications (red) and SNVs (brown) within

938 the TCGA cohort (top) and external validation datasets (bottom). 

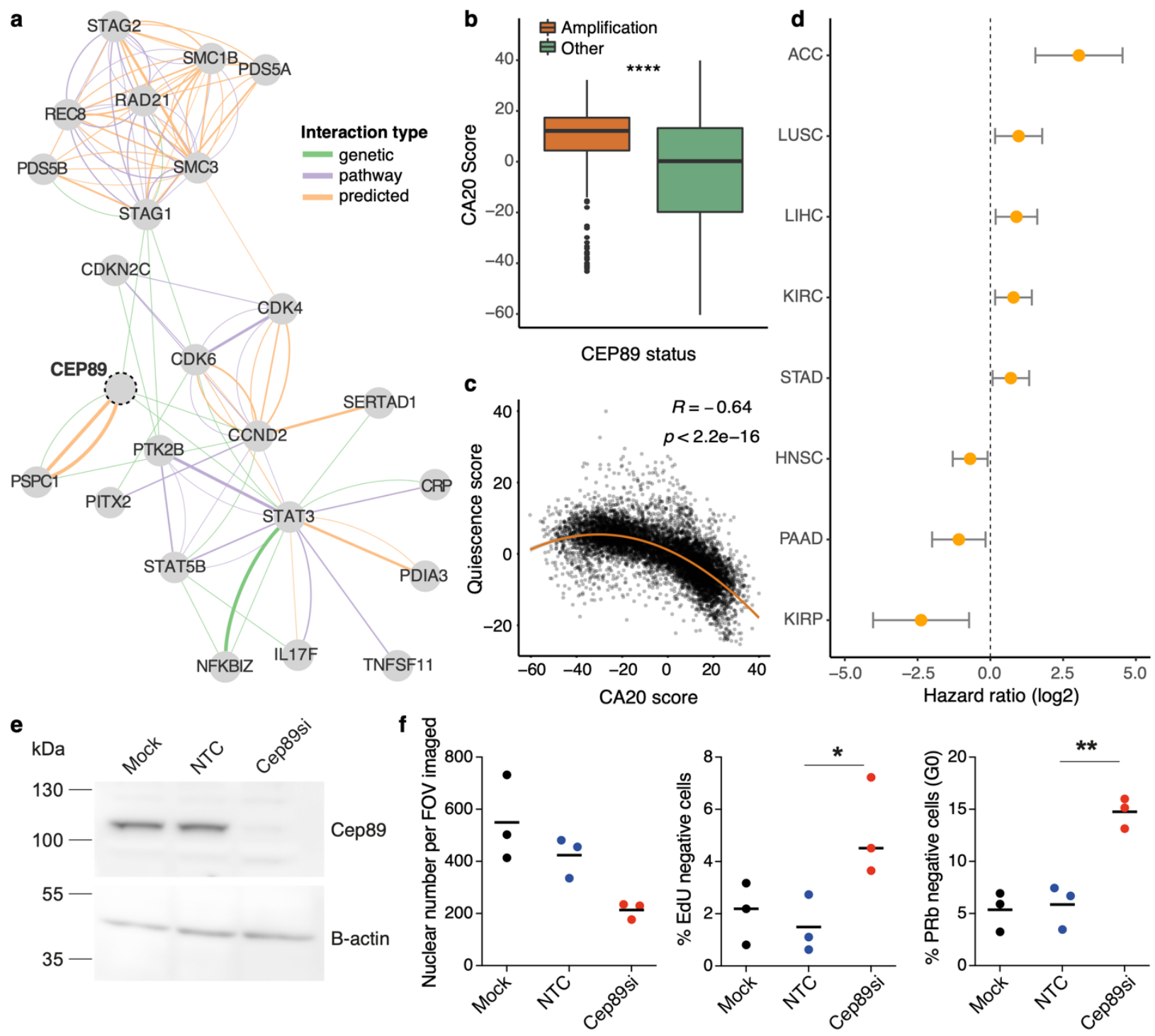

940 Figure 4: CEP89 amplification is associated with lower tumour dormancy. (a) Network

941 illustrating CEP89 interactions with cell cycle genes (from GeneMania). The edge colour indicates

942 the interaction type, with green representing genetic interactions, orange representing predicted

943 interactions and purple indicating pathway interactions. The edge width illustrates the interaction

944 weight. (b) CA20 scores are significantly increased in TCGA primary tumours containing a CEP89

945 amplification. (c) Pan-cancer relationship between CA20 scores and tumour quiescence across the

946 TCGA cohort. (d) Cox proportional hazards analysis estimates of the log hazards ratio for the

947 impact of CEP89 expression on patient prognosis within individual cancer studies, after adjusting

948 for tumour stage. Patients with high expression of CEP89 show significantly worse prognosis

949 within ACC, LUSC, LIHC, KIRC and STAD, but significantly better prognosis within HNSC, 
950 PAAD and KIRP studies. (e) Western blot showing depletion of Cep89 protein $48 \mathrm{~h}$ after siRNA

951 transfection of NCI-H1299 cells. Mock is lipofectamine only, NTC is Non-targeting control

952 siRNA. B-actin is used as a loading control. (f) Graphs show that Cep89 depletion in NCI-H1299

953 cells leads to a reduction in nuclear number and an increase in the fraction of quiescent cells,

954 measured by an increase in the percentage of EdU negative (24h EdU pulse) and Phospho-Ser

$955807 / 811 \mathrm{Rb}$ negative cells. One-Way ANOVA, ${ }^{*} \mathrm{p}<0.05,{ }^{* *} \mathrm{p}<0.01$. Mean of $\mathrm{n}=3$. 

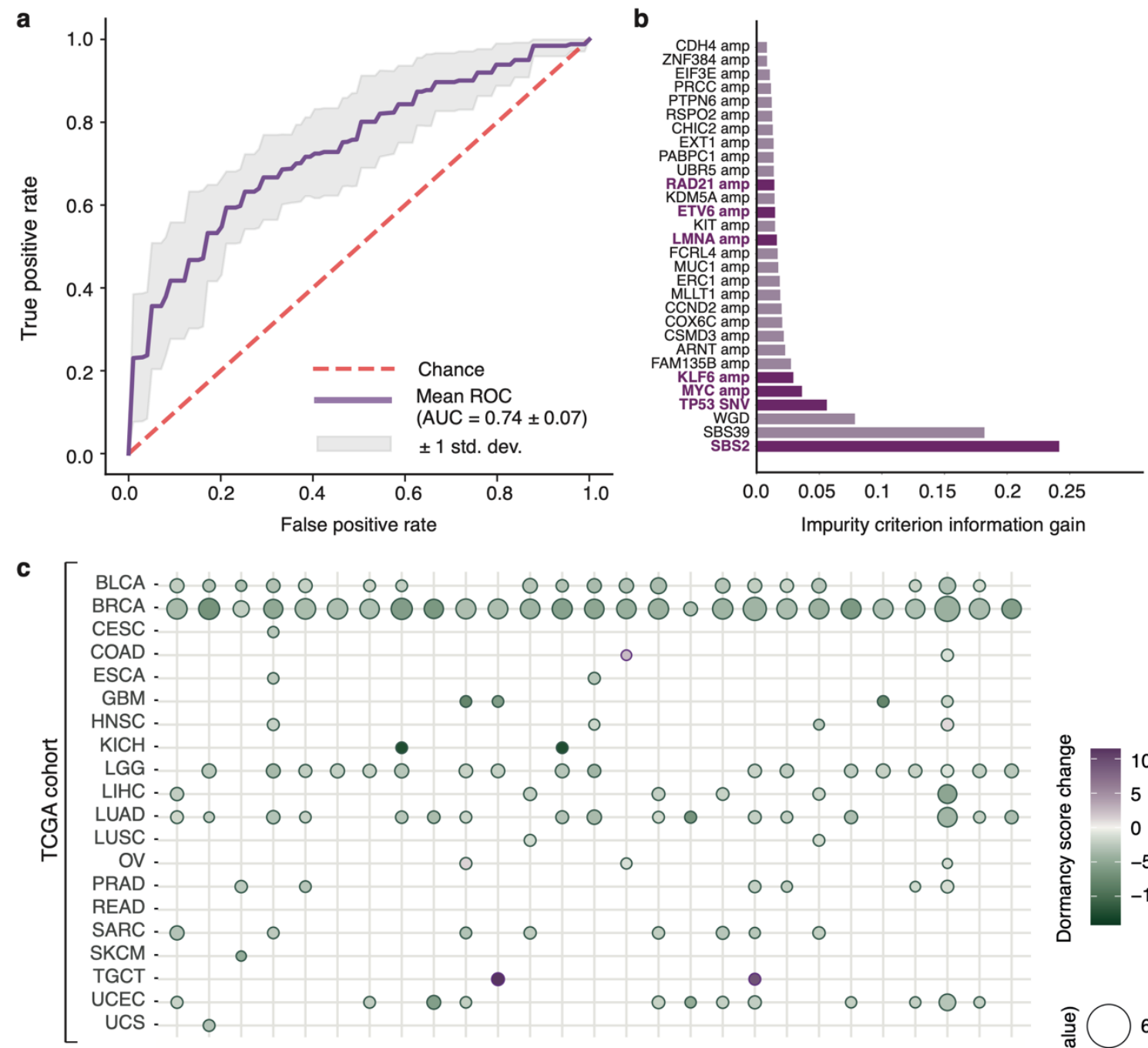

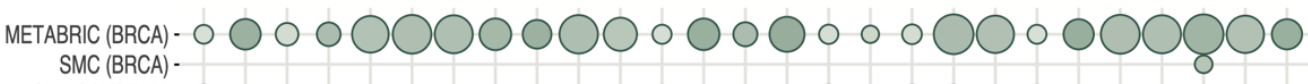 OncoSG (STAD) - MSKCC (BLCA) -
MSKCC (SARC) - O
TARGET (kidney) - 0

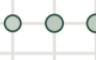

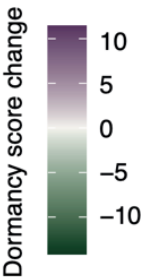

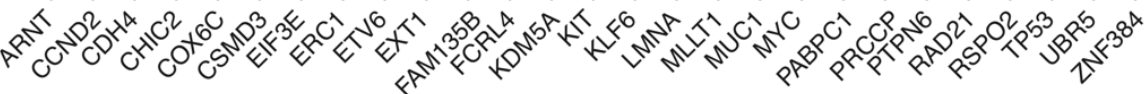

d

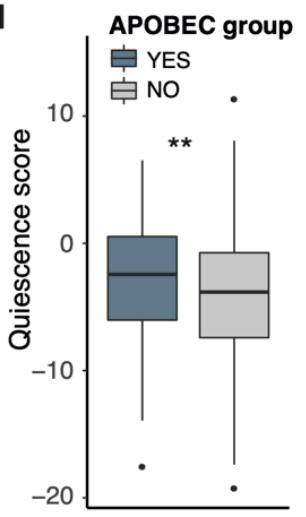

e

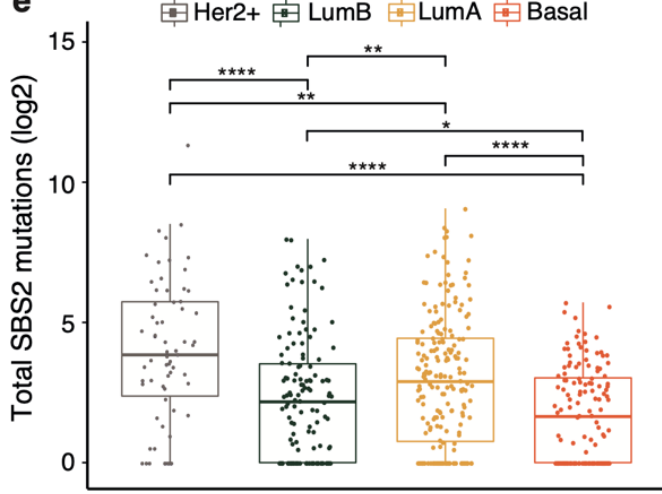

f

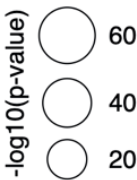

957 Figure 5: Genomic landscape of breast cancer dormancy. (a) ROC curve illustrating the mean 
randomisations. (b) Impurity criterion information gain for the top ranked genomic events

960 predictive of dormancy in the TCGA BRCA cohort, modelled using random forests. Features also

961 appearing in the pan-cancer model are highlighted in dark purple. WGD $=$ whole-genome

962 doubling. (c) Tissue-specific changes in dormancy estimates between samples with and without

963 dormancy-associated amplifications (red) and SNVs (purple), identified by the breast cancer

964 specific random forest model, within the TCGA cohort (top panel) and external validation datasets

965 (bottom panel). (d) Dormancy scores are significantly higher in APOBEC mutation-enriched breast

966 cancer samples. (e) APOBEC-linked SBS2 mutational burden compared between breast cancer

967 subtypes. (f) Association between dormancy scores, APOBEC enrichment and breast cancer

968 subtypes in TCGA.

969

970

971

972 

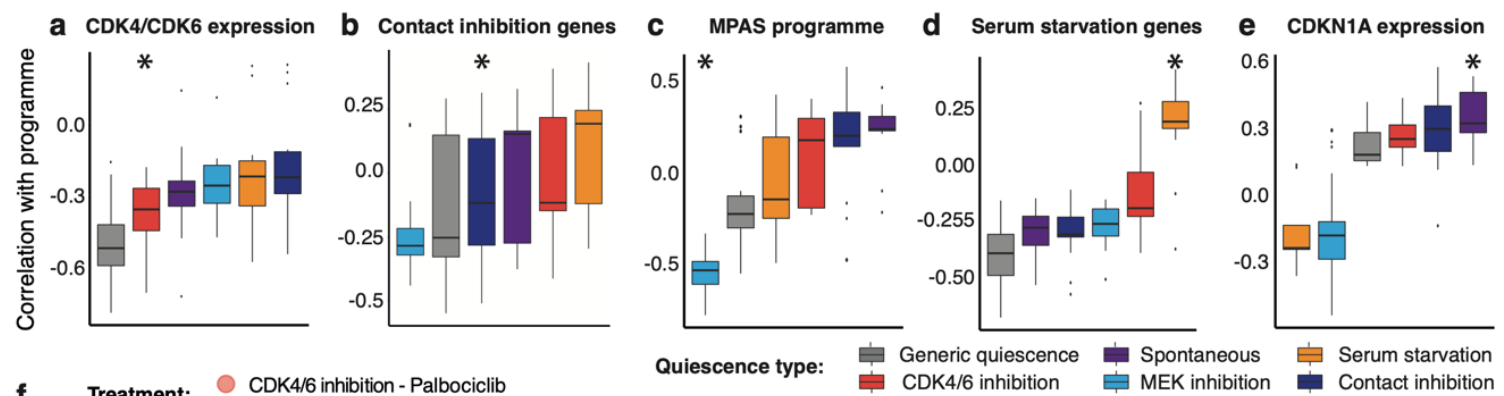

f Treatment: $\bigcirc$ CDK4/6 introl
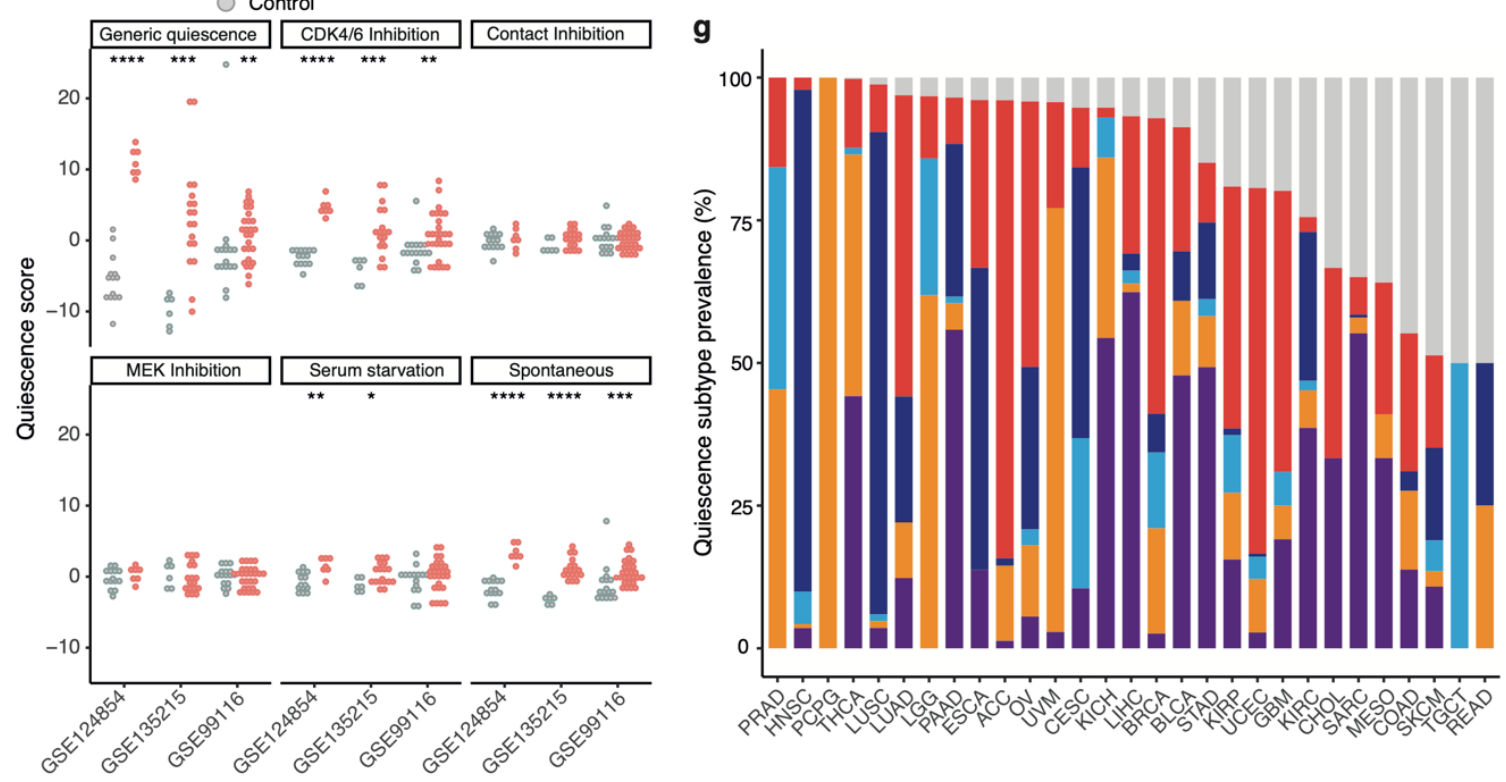

974 Figure 6: Pan-cancer characterisation of individual quiescence programmes. (a-e)

975 Comparison of correlation coefficients between quiescence programme scores and (a) mean

expression of CDK4 and CDK6, (b) mean expression of curated contact inhibition genes, (c) a

transcriptional MAPK Pathway Activity Score (MPAS), (d) mean expression of curated serum

starvation genes, and (e) CDKN1A expression, across TCGA cancers. The correlations expected to

be strongest (either negative or positive) are denoted by an asterisk. The generic quiescence score refers to scores calculated using the original list of 139 genes differentially expressed across all 5

981 forms of quiescence. (f) Comparison of quiescence programme scores measured in cancer cell lines before (grey) and after (red) Palbociclib treatment across three validation studies. Datasets used for quiescence type composition of samples estimated to be dormant across individual cancer types. The same colour legend as in (a) is applied. Gray bars represent the proportion of samples for which the quiescence type could not be estimated. 

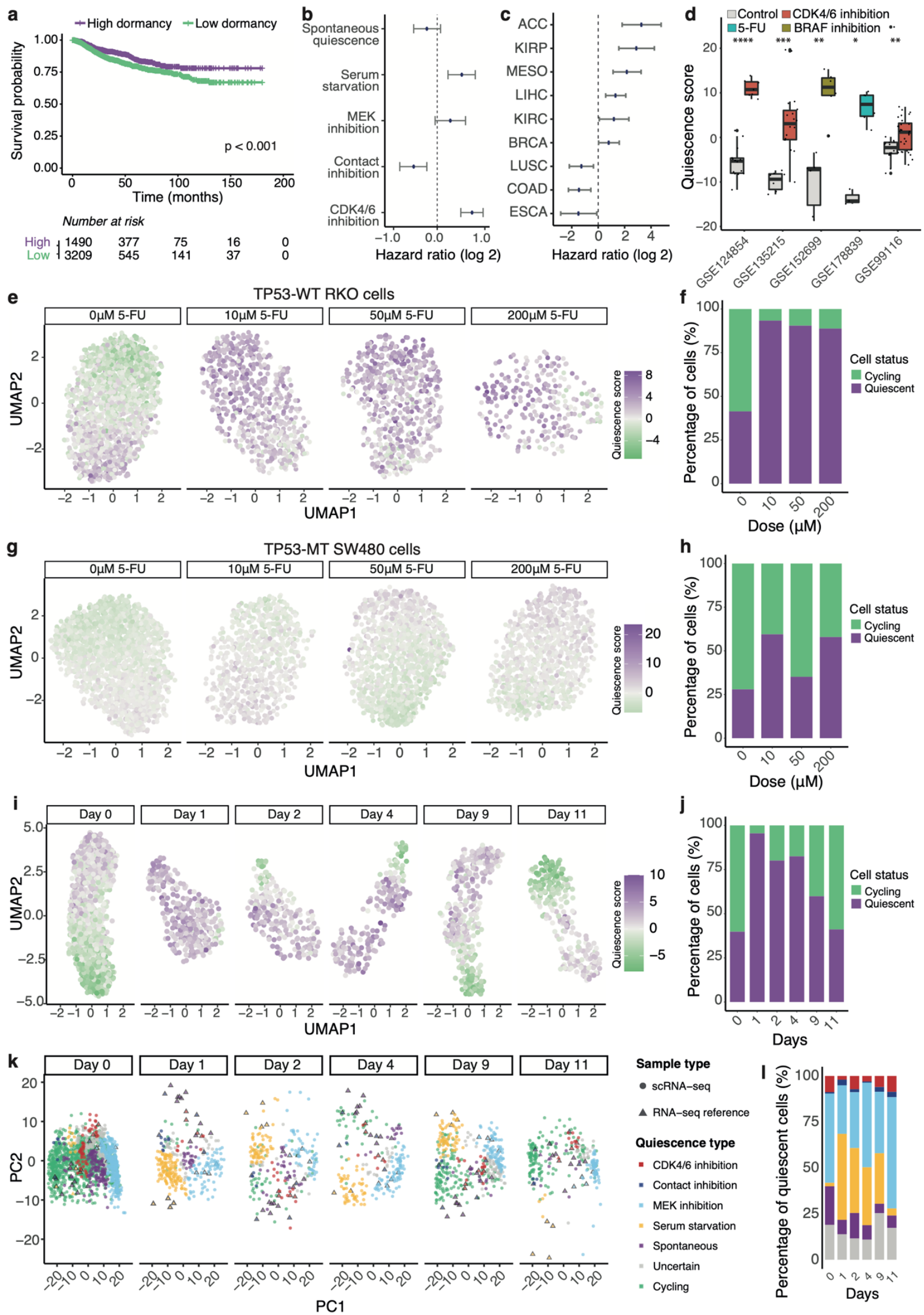

Figure 7: Impact of cellular dormancy on patient prognosis and treatment response. (a)

Disease-specific survival based on tumour dormancy for patients from TCGA within 15 years of 
990 follow up. Patients with high primary tumour dormancy showed significantly better prognosis than

991 patients with low dormancy. (b-c) Hazard ratio ranges illustrating the impact of different forms of

992 quiescence (b) and different tissues (c) on patient prognosis, after taking into account potential

993 confounding factors. Values above 0 indicate significantly better prognosis in the context of high

994 tumour dormancy. (d) Change in quiescence scores inferred from bulk RNA-seq across breast,

995 pancreatic, colorectal and skin cancer cells in response to treatment with the CDK4/6 inhibitor

996 Palbociclib, 5-FU or the BRAF inhibitor Vemurafenib. (e-f) UMAP plot illustrating the response of

997 the TP53-proficient RKO colorectal cancer cell line to various 5-FU doses and the corresponding

998 proportions of cells predicted to be quiescent/proliferating. Each dot is an individual cell, coloured

999 according to its dormancy level. (g-h) The same as previous, but for the TP53-deficient SW480

1000 cell line. (i-j) UMAP plot illustrating the response of individual PC9 NSCLC cells to the EGFR

1001 inhibitor Erlotinib across several time points and the corresponding proportion of cells predicted to

1002 be quiescent/proliferating. (k) Principal component analysis illustrating the superimposition of 1003 scRNA-seq profiles (circles) of quiescent NSCLC cells before/after EGFR inhibition onto the bulk 1004 RNA-seq reference data (triangles) for MCF10A cells occupying various quiescence states. (I) The 1005 proportion of NSCLC cells in (k) predicted to occupy different quiescence states across several 1006 time points. 
a
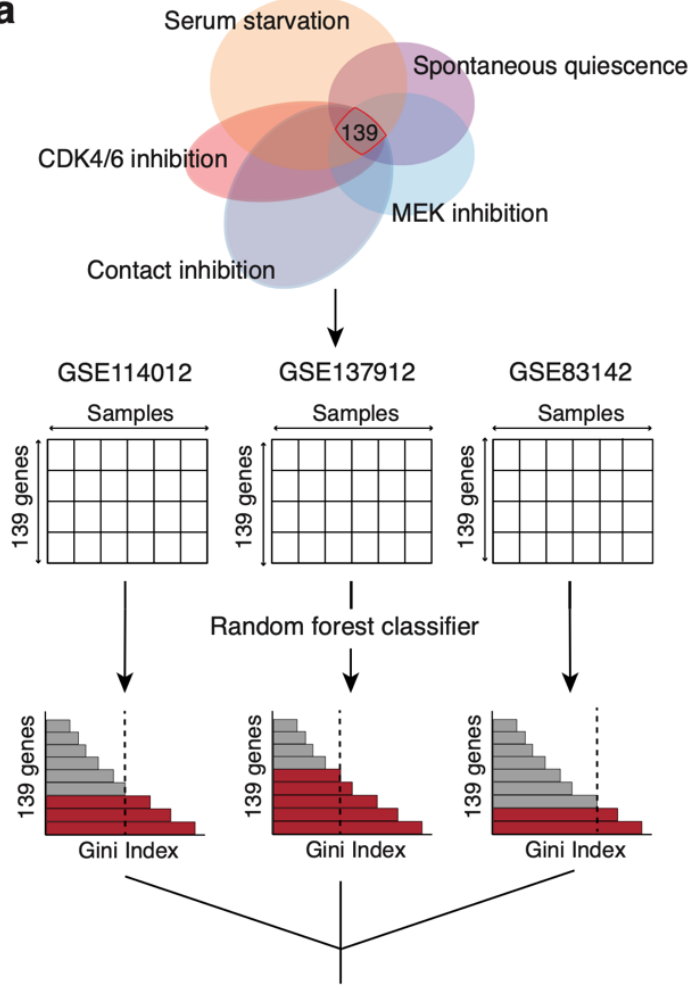

Gini Index thresholding

Refined signature of quiescence b
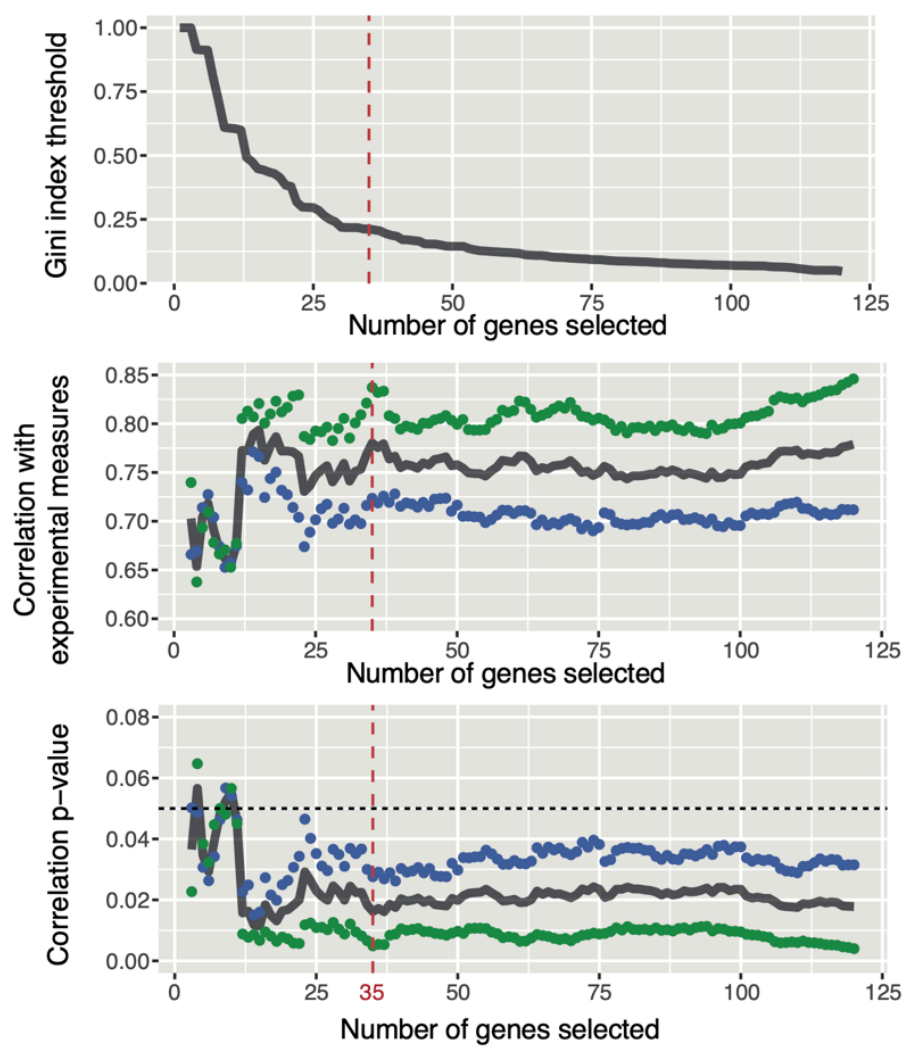

Cell line quiescence level
measurement:

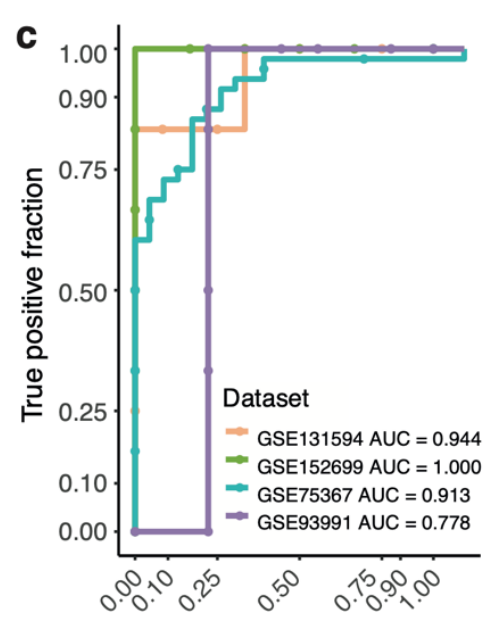

False positive fraction
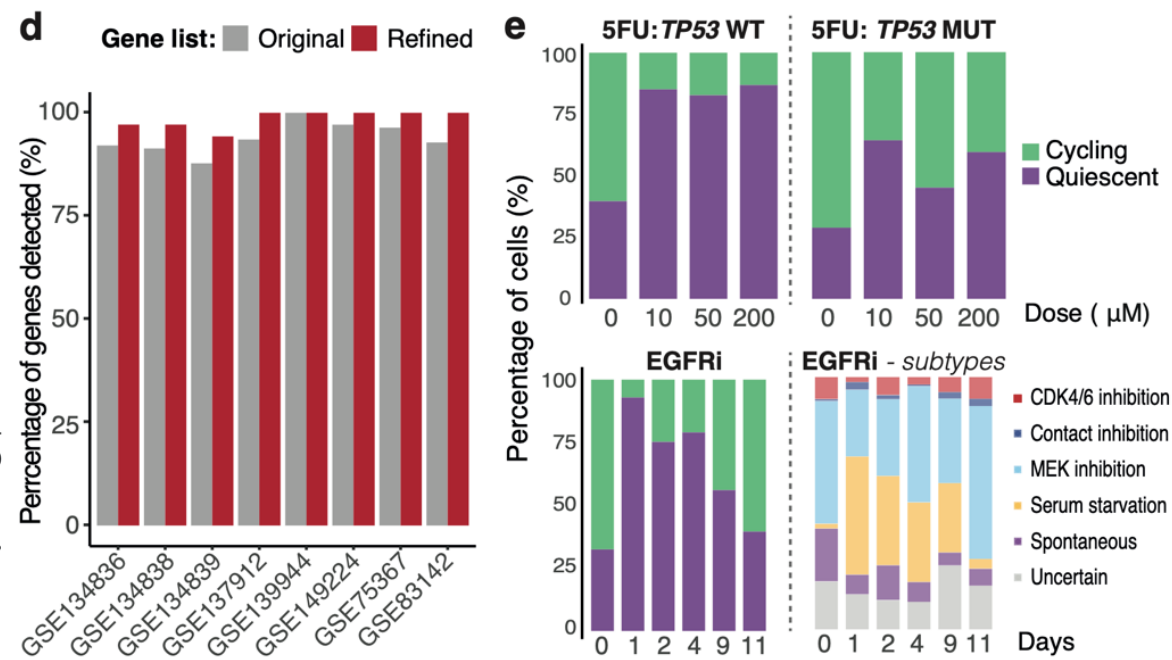

Figure 8: Optimisation of dormancy signature for use in scRNA-seq data. (a) Methodology for

refining the gene signature of dormancy: random forest classifiers are trained to distinguish dormant from cycling tumours on three high confidence datasets; Gini index thresholding is optimised to prioritise a final list of 35 genes. (b) Gini index variation, correlation with experimentally measured quiescence via EdU and PRb staining assays, and corresponding p-values are plotted as the number of genes considered in the model is increased. The red dotted line 
1014 indicates the threshold chosen for the final solution of 35 genes. The black dotted line indicates the

1015 threshold for p-value significance. (c) Additional external validation of the 35 gene signature acting

1016 as a classifier of quiescent and proliferation cells in single cell and bulk datasets. (d) Dropout in

1017 single cell data by gene signature. The percentage of genes out of the 35 (red) and 139 (grey) gene

1018 lists with reported expression across the single-cell RNA-seq datasets analysed in this study. (e)

1019 Proportion of cycling and quiescent cells estimated in single cell datasets of p53 wild type and

1020 mutant lines treated with 5FU, as well as cells treated with EGFR inhibitors. Data as in Figure 7. 
a

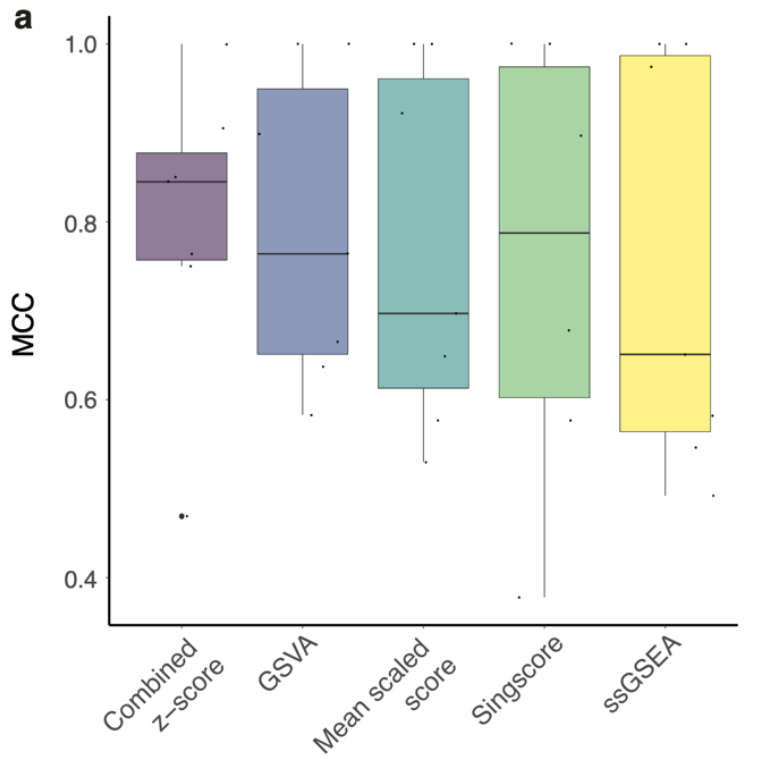

b

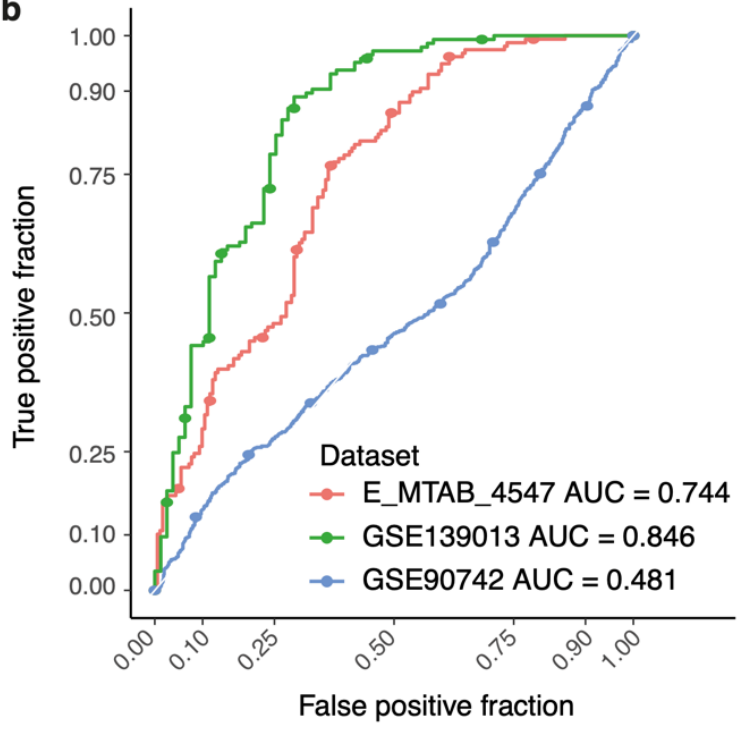

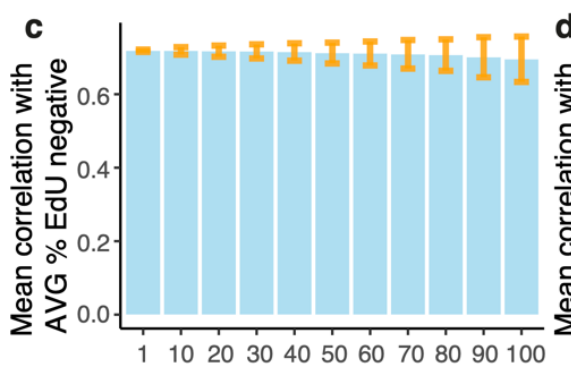

Number of genes removed

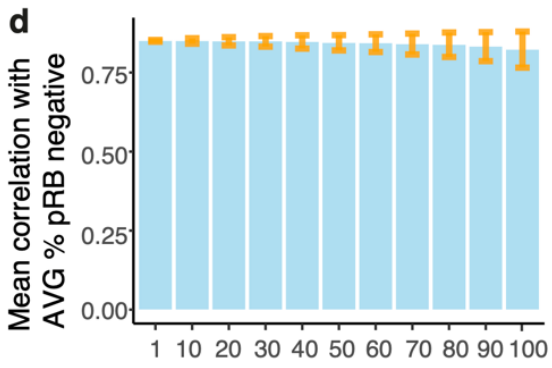

Number of genes removed

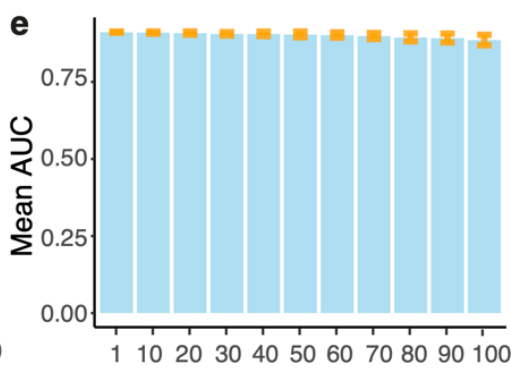

Number of genes removed

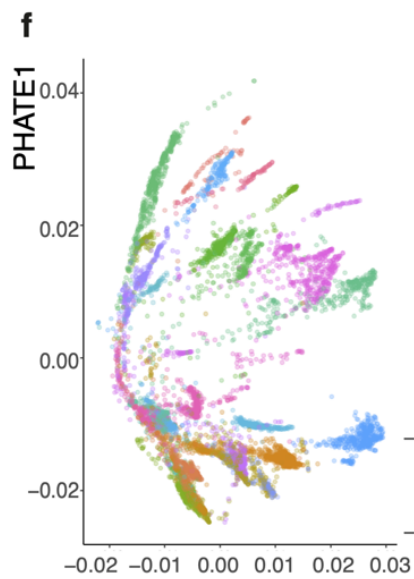

g
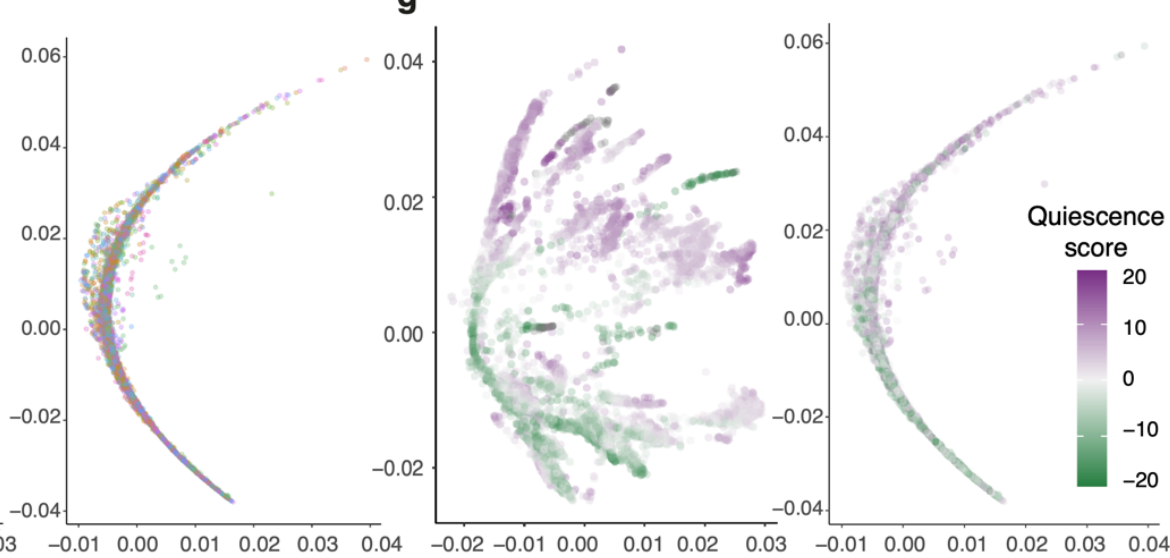

PHATE2

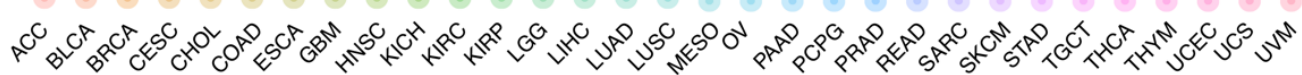

Extended Data Figure 1: Quiescence evaluation and validation. (a) Classification accuracies

measured using the Matthews correlation coefficient (MCC) comparing the combined z-score

quiescence scoring method to other scoring methodologies across the seven single-cell and bulk

RNA sequencing validation datasets. (b) Receiver operating characteristic (ROC) curves 
1028 illustrating the accuracy of the combined z-score methodology when separating dormant and

1029 proliferating hematopoietic stem cells from three publicly available single-cell RNA-seq datasets.

1030 Datasets used are denoted by their corresponding GEO series accession number and the area under

1031 the curve (AUC) for each dataset is specified. (c) Mean correlation between computationally

1032 inferred dormancy scores after randomly removing variable number of genes from the dormancy

1033 signature and the fraction of cells entering quiescence in nine lung adenocarcinoma cell lines, as

1034 assessed through an EdU staining assay. (d) Same as (c) but with experimental levels of quiescence

1035 measured using a phospho-Rb staining assay. (e) Mean classification accuracy across 7 bulk and

1036 single-cell RNA-seq validation datasets after randomly removing variable number of genes from

1037 the dormancy signature. (f-g) PHATE plots displaying the first two principal components obtained

1038 from dimensionality reduction analysis across 8,005 primary tumour TCGA samples, based on the

1039 expression of 139 genes differentially expressed across different quiescence programmes before

1040 (left) and after (right) removal of tissue specific expression patterns. Each primary tumour sample

1041 is coloured by their corresponding tissue of origin (f) or the corresponding quiescence score (g). 

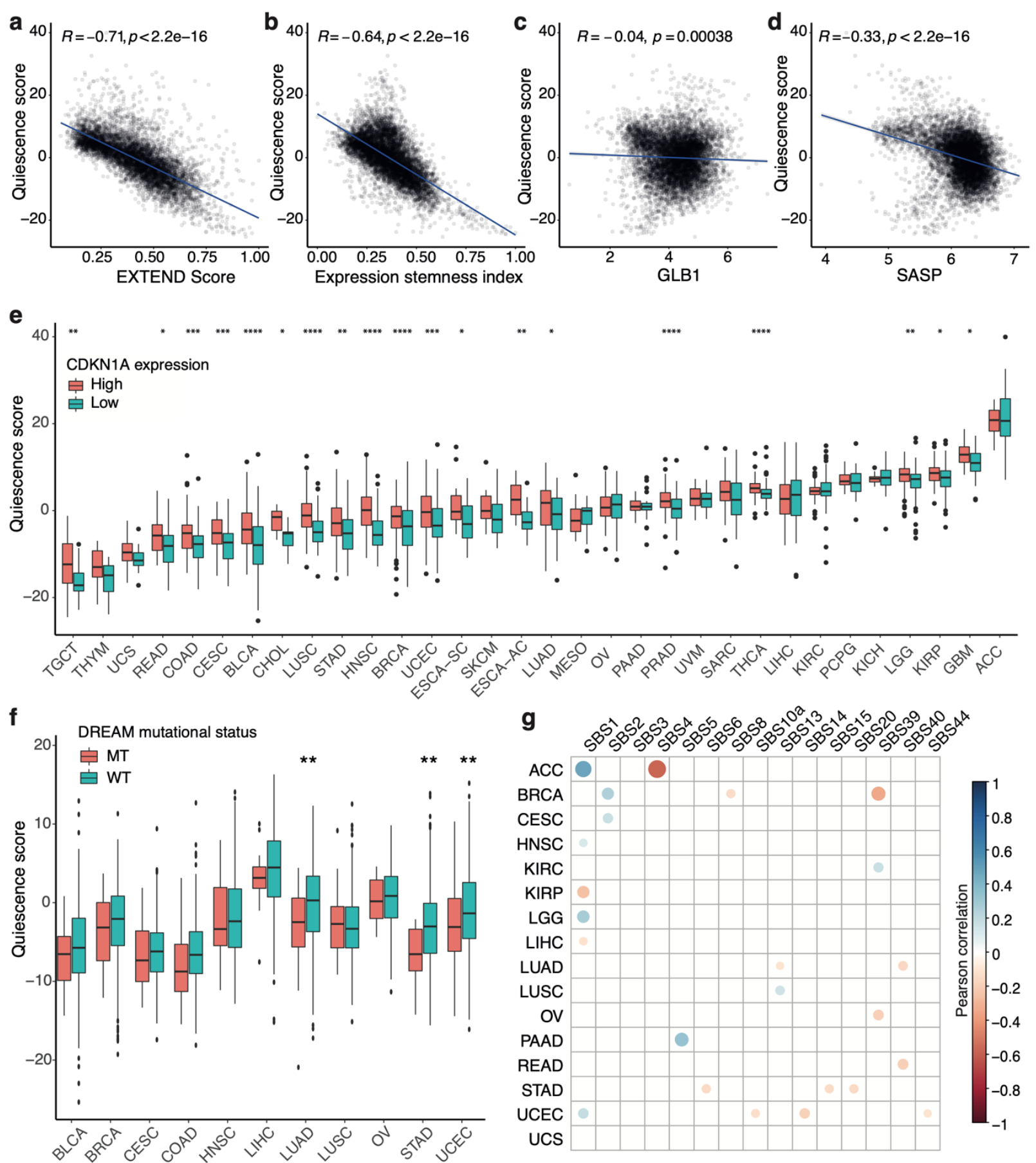

Extended Data Figure 2: Expression and genomic patterns of dormant tumours. (a-d)

1044 Correlation between quiescence scores and (a) telomerase activity inferred via the "EXTEND" score, (b) stemness expression index, (c) the expression of the $\beta$ galactosidase-encoding gene GLB1 and (d) the expression of the Senescence-Associated Secretory Phenotype (SASP) gene expression. (e) Consistently higher levels of quiescence are detected in samples with high 
bioRxiv preprint doi: https://doi.org/10.1101/2021.11.12.468410; this version posted December 3, 2021. The copyright holder for this preprint (which was not certified by peer review) is the author/funder, who has granted bioRxiv a license to display the preprint in perpetuity. It is made available under aCC-BY-NC-ND 4.0 International license.

1049 samples with low expression (lower quartile of the expression distribution) across TCGA cancer

1050 types. (f) Consistently higher levels of dormancy are detected in samples with a functional

1051 DREAM complex. TCGA studies where mutations within one of the DREAM components could

1052 be detected in at least 10 patients are shown. (g) Correlation between quiescence levels and

1053 mutational signature contributions across TCGA cancer types. Only significant correlations are

1054 displayed (Pearson correlation p-value $<0.05)$ 
bioRxiv preprint doi: https://doi.org/10.1101/2021.11.12.468410; this version posted December 3, 2021. The copyright holder for this

preprint (which was not certified by peer review) is the author/funder, who has granted bioRxiv a license to display the preprint in perpetuity. It is made available under aCC-BY-NC-ND 4.0 International license.

a

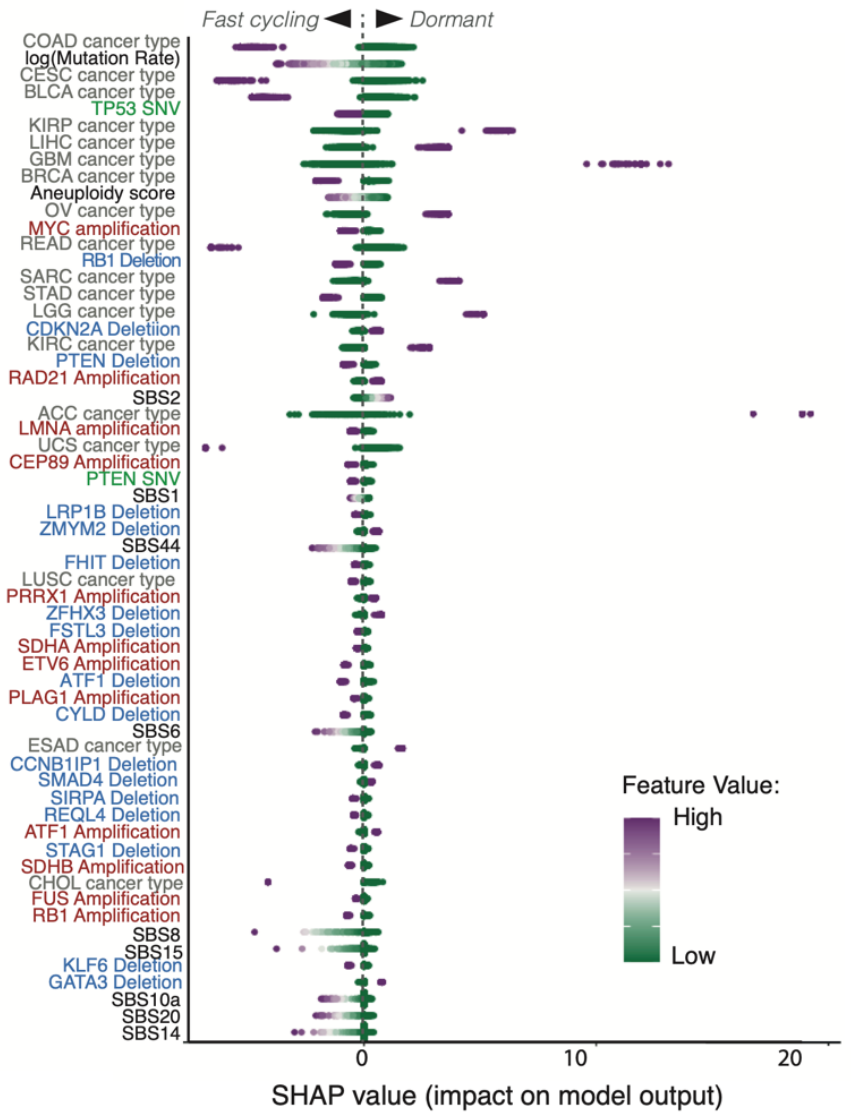

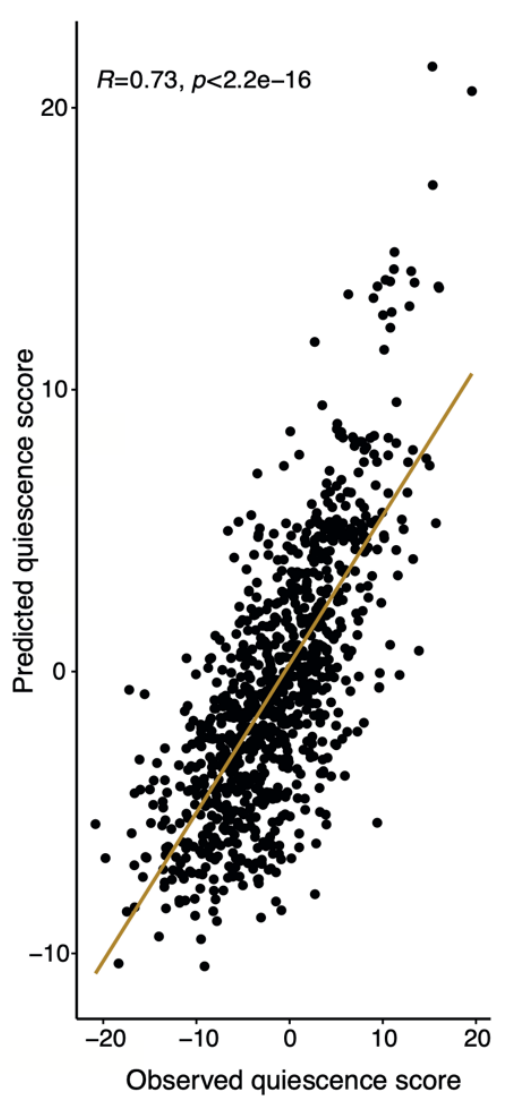

c G1 Phase

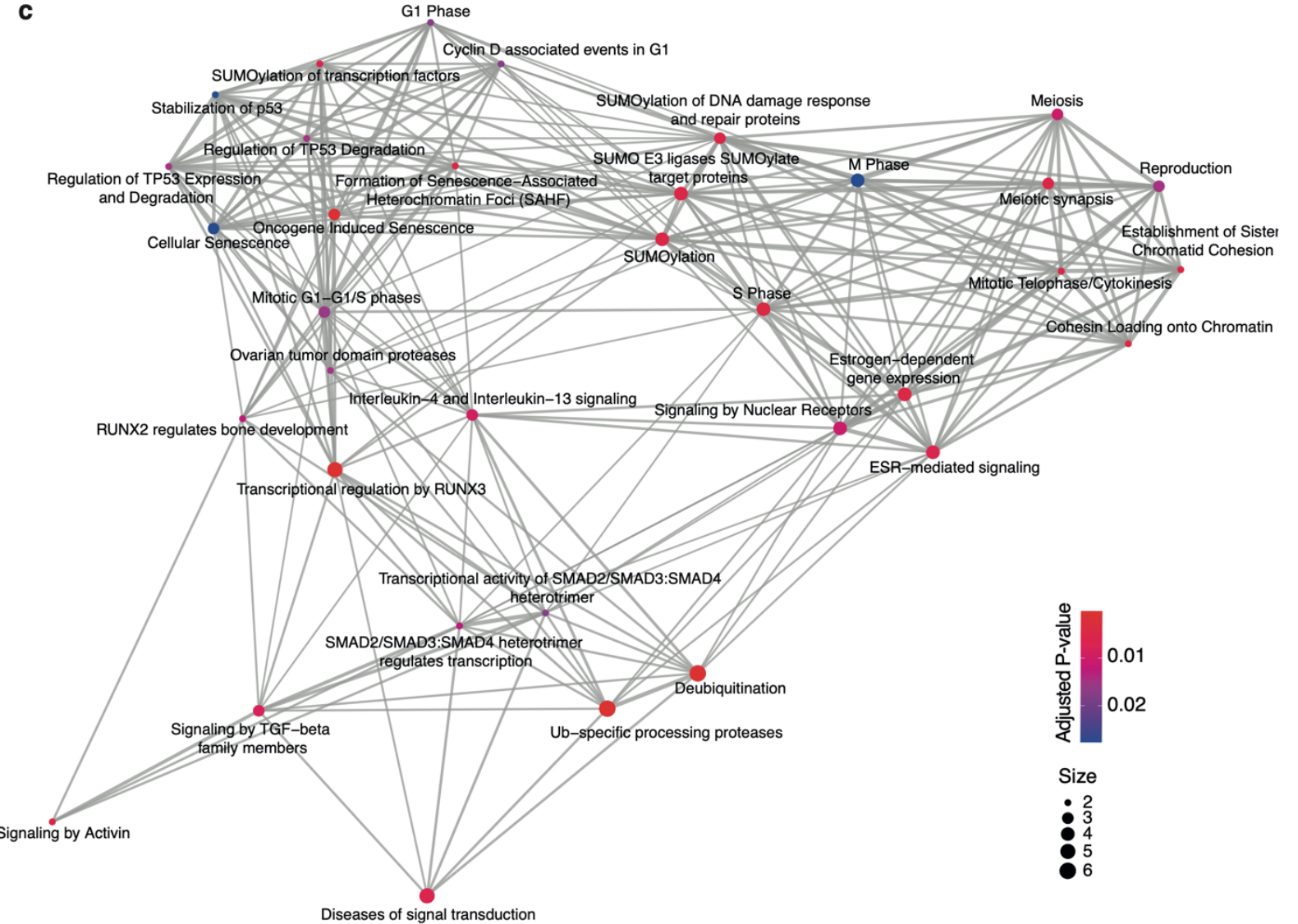

1057 Genomic features and cancer types significantly associated with dormancy, ranked according to 

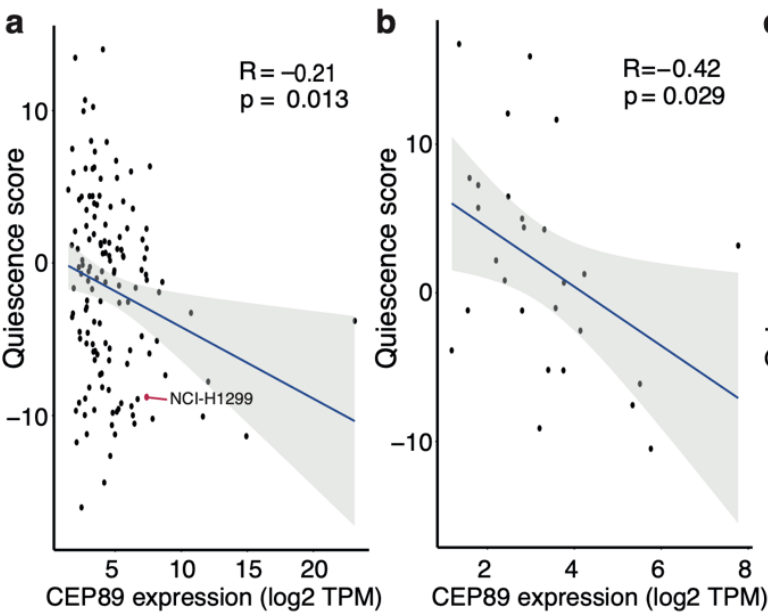

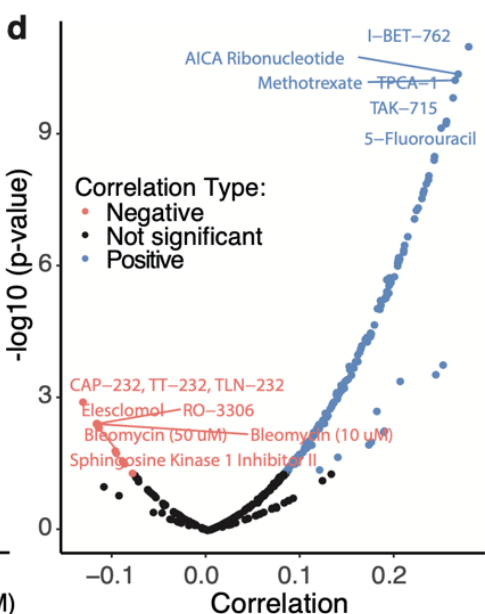

their importance in the pan-cancer linear regression model (from highest to lowest contribution).

The genomic features highlighted are identical to Figure $3 \mathrm{c}$, but cancer types are shown here as well. Each point corresponds to one sample and is coloured according to the original value of the respective feature. For discrete variables purple indicates the presence of the feature and green indicates the absence of the feature. For cancer type variables, purple indicates the patient belongs to the specified TCGA cancer type group. The Shapley values indicate whether the quiescence scores predicted by the ensemble elastic net regression model for each patient are increased or decreased depending on the cancer type of each individual as well as specific SNVS, CNV and other genomic features. (b) Correlation between the observed quiescence scores within the test dataset (x axis) and scores predicted by the linear regression model using the selected pan-cancer features (y axis). (c) Pathways significantly altered in association with tumour dormancy.

\section{Extended Data Figure 4: CEP89 expression is associated with quiescence and has prognostic}

value. (a-c) CEP89 expression is negatively correlated with quiescence scores in lung (a), pancreas

(b) and thyroid (c) cancer cell lines. The NCI-H1299 cell line where CEP89 activity was tested is expression. The volcano plot shows the Pearson correlation coefficients and the corresponding pvalues for associations between CEP89 expression and Area Under the dose-response Curve (AUC) for each compound across cell lines form the CCLE. 
bioRxiv preprint doi: https://doi.org/10.1101/2021.11.12.468410; this version posted December 3, 2021. The copyright holder for this

preprint (which was not certified by peer review) is the author/funder, who has granted bioRxiv a license to display the preprint in perpetuity. It is made available under aCC-BY-NC-ND 4.0 International license.

a

CDK4/CDK6 expression

Generic quiescence score

Contact inhibition score

CDK4/6 inhibition score

MEK inhibition score

Serum starvation score

Spontaneous quiescence score

$$
-0.79
$$

ষ্ঠ 焉

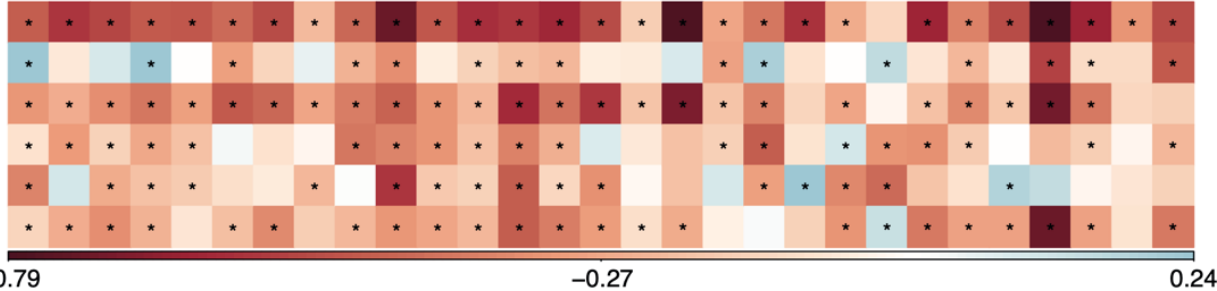

b

Contact inhibition genes

Generic quiescence score

Contact inhibition score CDK4/6 inhibition score MEK inhibition score Serum starvation score Spontaneous quiescence score

离 焉

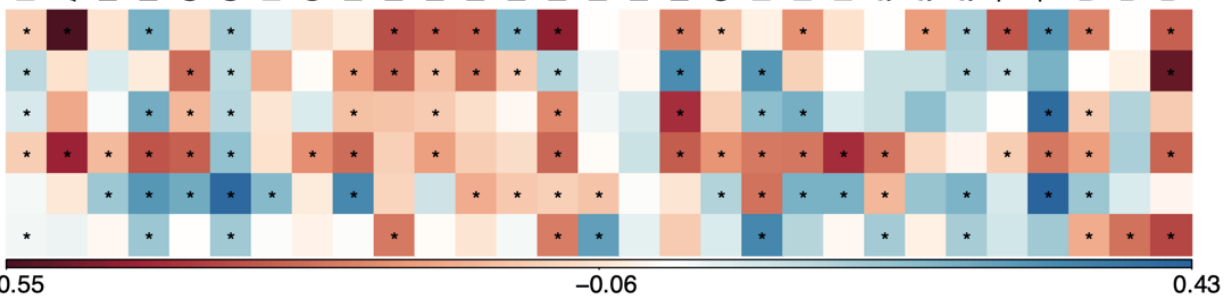

c

MPAS scores

্ֻঠ

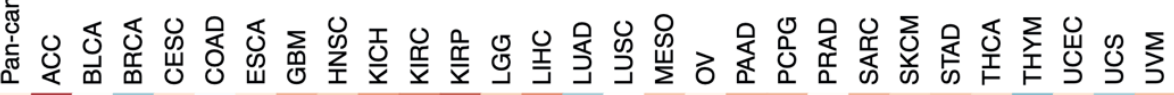

Generic quiescence score

Contact inhibition score CDK4/6 inhibition score

MEK inhibition score

Serum starvation score Spontaneous quiescence score

$$
-0.78
$$
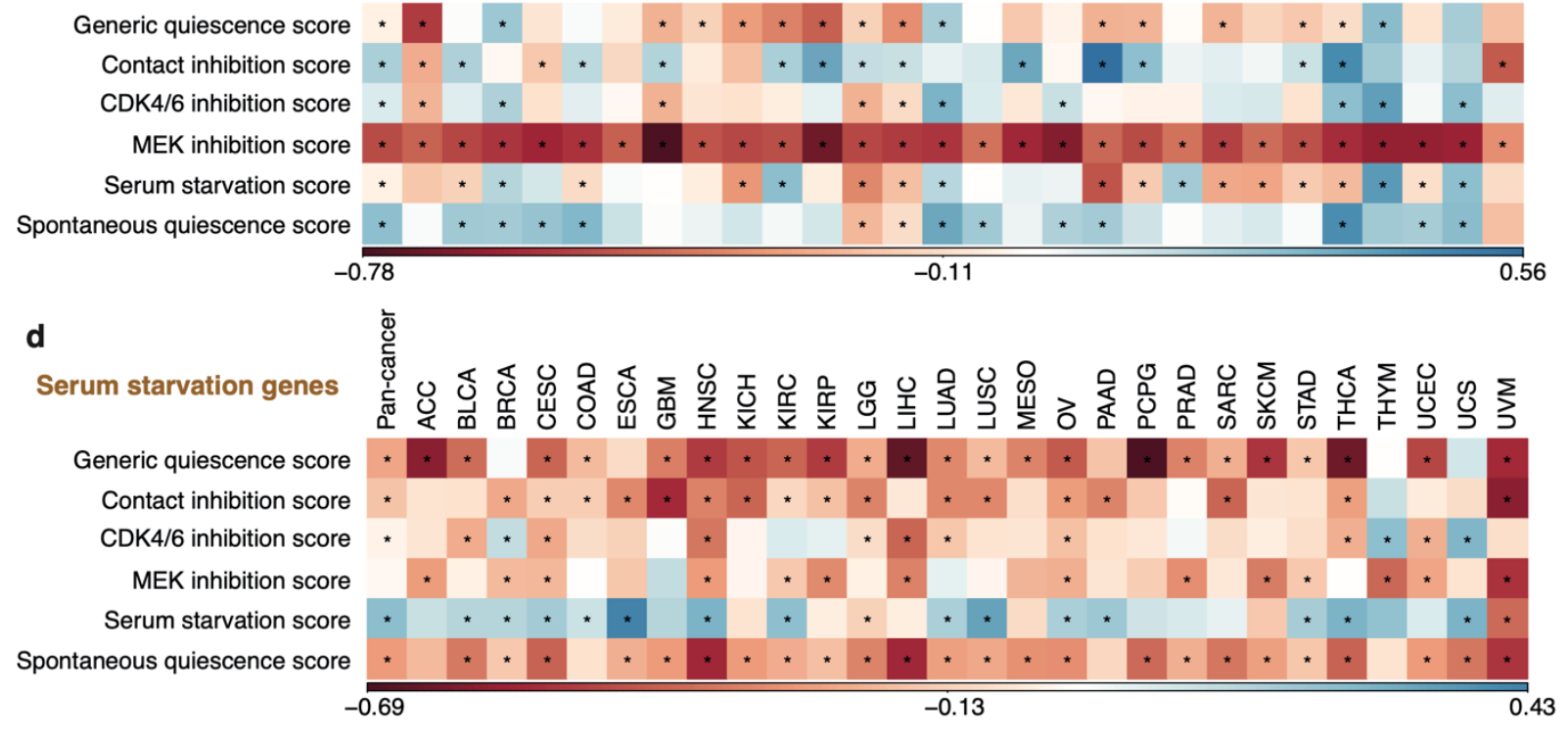

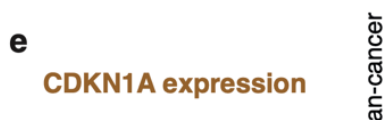
,

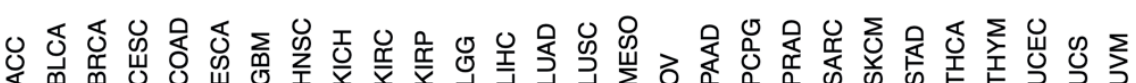

Generic quiescence score Contact inhibition score CDK4/6 inhibition score MEK inhibition score Serum starvation score Spontaneous quiescence score

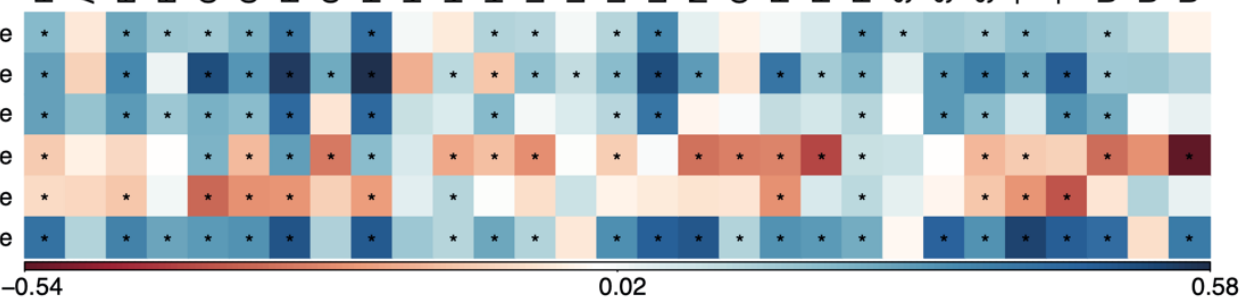

1079 Extended Data Figure 5: Quiescence subtype validation. Correlation between subtype-specific 
bioRxiv preprint doi: https://doi.org/10.1101/2021.11.12.468410; this version posted December 3, 2021. The copyright holder for this preprint (which was not certified by peer review) is the author/funder, who has granted bioRxiv a license to display the preprint in perpetuity. It is made available under aCC-BY-NC-ND 4.0 International license.

1081 contact inhibition genes, (c) MPAS scores, (d) mean expression of curated serum starvation genes 1082 and (e) CDKN1A expression, across individual TCGA cancer studies and pan-cancer. The colour 1083 gradient indicates the strength of the association (positive or negative). 
a
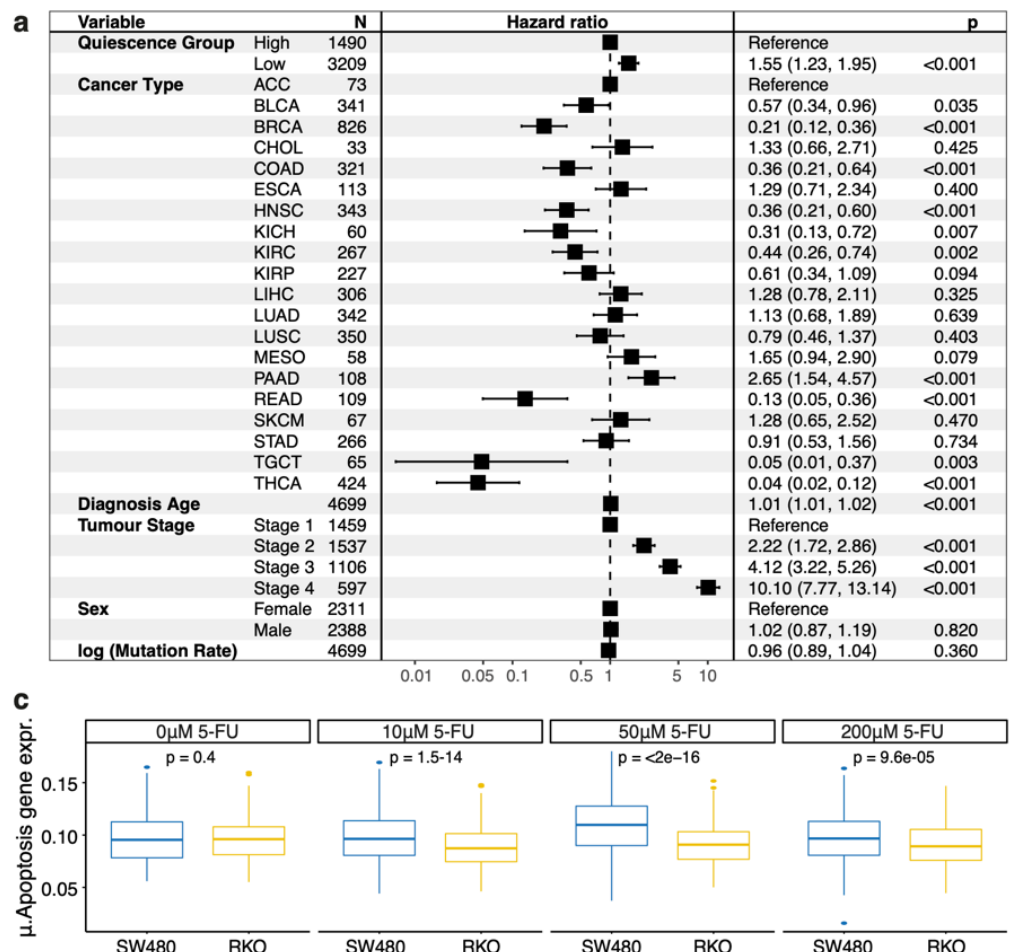

b 皁 Stage 1 追 Stage 2

官 Stage 3 帛 Stage 4
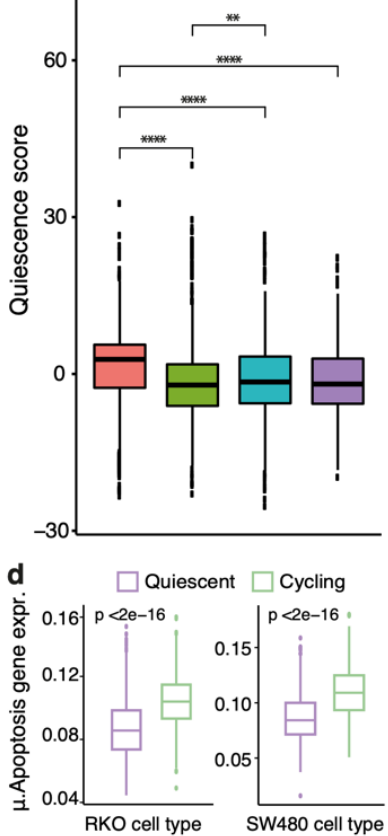

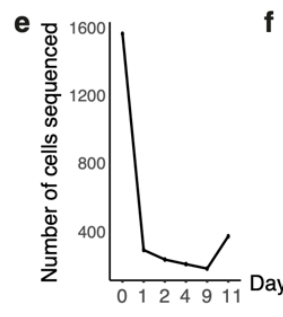

g

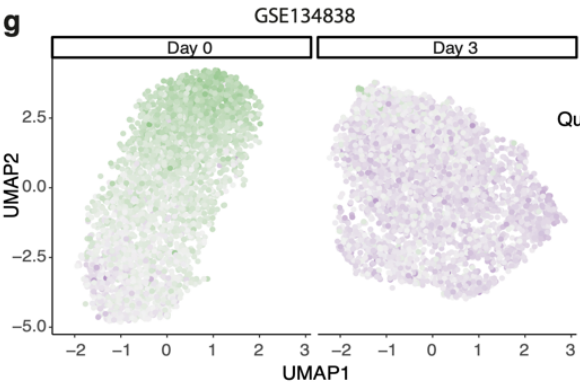

i
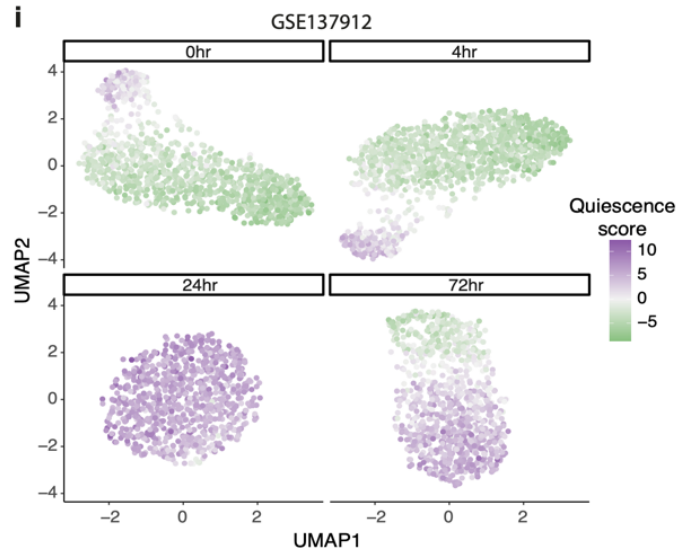

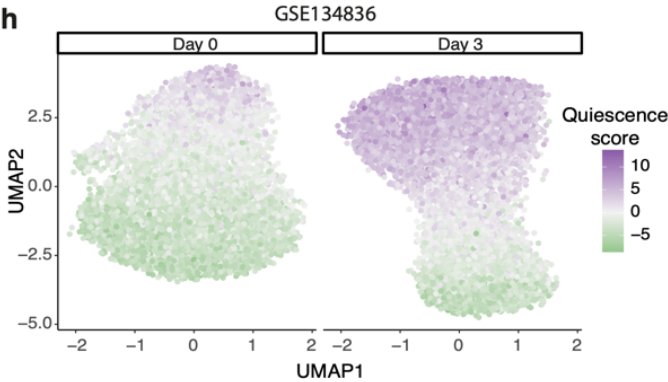

j

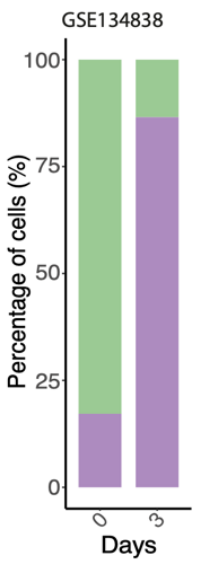

GSE134836

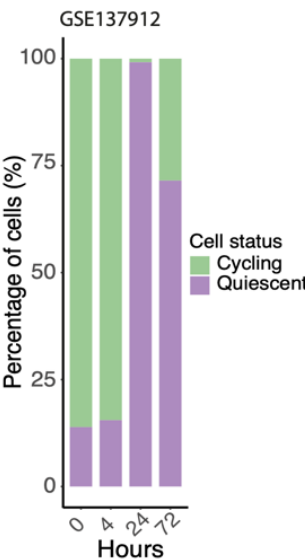

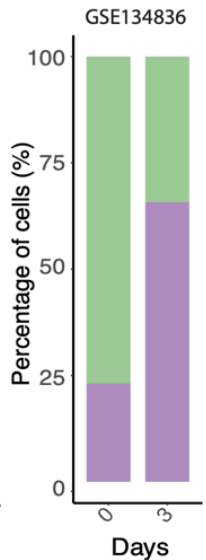

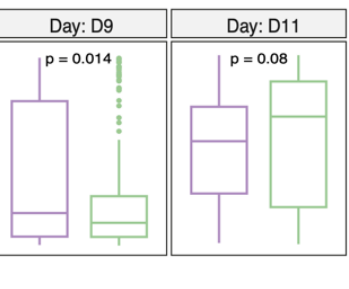

Extended Data Figure 6: Tumour dormancy relevance to clinical outcome. (a) Multivariate 
on tumour dormancy (binarized by optimised survival cut-off into high, where score $>2.95$; and low, where score $<=2.95$ ) and potential cofounding factors. (b) Quiescence scores in TCGA cancers compared across tumour stages. (c) The average expression of apoptosis genes compared between TP53 wild type RKO and TP53 mutant SW480 colorectal cancer cell lines in response to various 5-FU treatment doses. (d) The average expression of apoptosis genes compared between quiescent and cycling cells within the TP53 WT RKO and TP53 MT SW480 cell lines. (e) The number of NSCLC cells sequenced during the EGFR inhibition treatment time course. (f) The average expression of apoptosis genes compared between quiescent and cycling cells at different time points during EGFR inhibition treatment. There is a notable increase in apoptotic activity of cycling cells at day 1. (g) Response of melanoma cancer cells to BRAF inhibition treatment across several time points, illustrated using UMAP plots coloured by quiescence score of individual cells.

(h) Response of NSCLC cells to EGFR inhibition via Erlotinib across several time points,

1099 illustrated using UMAP plots coloured by quiescence score of individual cells. (i) Response of lung 1100 adenocarcinoma cell lines to KRAS inhibition treatment across several time points, illustrated 1101 using UMAP plots coloured by quiescence score of individual cells. (j) Percentage of cells 1102 illustrated in (g-i) predicted to be quiescent across the treatment time course. 
bioRxiv preprint doi: https://doi.org/10.1101/2021.11.12.468410; this version posted December 3, 2021. The copyright holder for this

preprint (which was not certified by peer review) is the author/funder, who has granted bioRxiv a license to display the preprint in perpetuity. It is made available under aCC-BY-NC-ND 4.0 International license.

a

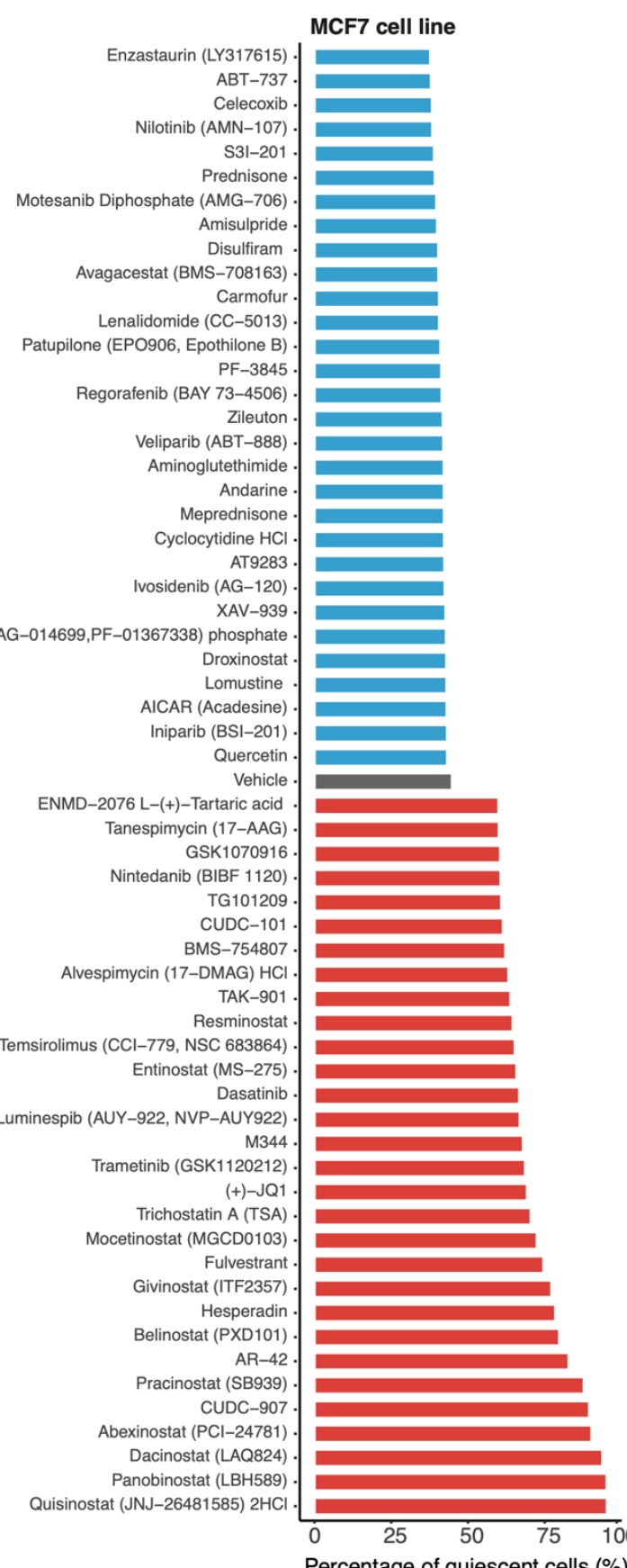

Percentage of quiescent cells $(\%)$

\section{MCF7 cell line}

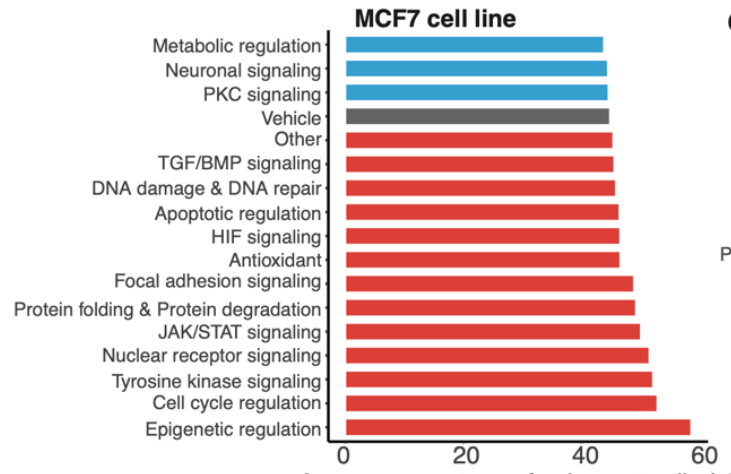

Average percentage of quiescent cells (\%) b

Enzastaurin (LY317615)
Patupilone (EPO906, Epothilone B)
Tie2 kinase inhibitor
YM155 (Sepantronium Bromide)
Disulfiram
Andarine
Meprednisone

Roscovitine (Seliciclib,CYC202) Motesanib Diphosphate (AMG-706) RG108 Nilotinib (AMN-107) Azacitidine Linifanib (ABT-869) Aminoglutethimide Quercetin Amisulpride Raltitrexed S3I-201

Baricitinib (LY3009104, INCB028050) ABT-737 Lenalidomide (CC-5013) Prednisone Mesna Costunolide Streptozotocin (STZ) Sodium Phenylbutyrate Celecoxib Fasudil (HA-1077) HCI Avagacestat (BMS-708163)

apatinib (GW-572016) Danusertib (PHA-739358) UDC-101 SL-327 Resminostat BMS-754807 ENMD-2076 L-(+)-Tartaric acid MLN8054 Dasatinib SNS-314 Pirarubicin Alisertib (MLN8237) PHA-680632 Mocetinostat (MGCD0103) AMG-900 TAK-901

Barasertib (AZD1152-HQPA) Givinostat (ITF2357) GSK1070916 Belinostat (PXD101) Triamcinolone Acetonide Pracinostat (SB (SB939) Hesperadin Abexinostat ( $\mathrm{PCl}-24781)$ Dacinostat (LAQ824) CUDC-907

Panobinostat (LBH589) Quisinostat (JNJ-26481585) 2 $\mathrm{HCl}$. Trametinib (GSK1120212)

d A549 cell line

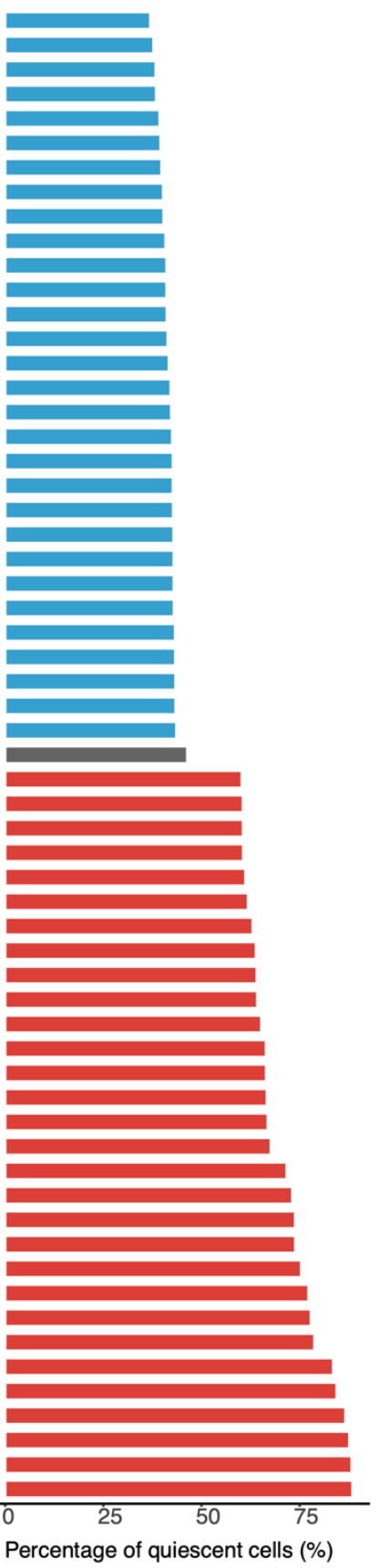

A549 cell line

Antioxidant esion signaling PKC signaling Metabolic regulation Neuronal signaling Vehicle TGF/BMP signaling JAK/STAT signaling Protein folding \& Protein degradation DNA damage \& DNA repair Nuclear receptor signaling Apoptotic regulation HIF signaling

Tyrosine kinase signaling Epigenetic regulation Cell cycle regulation

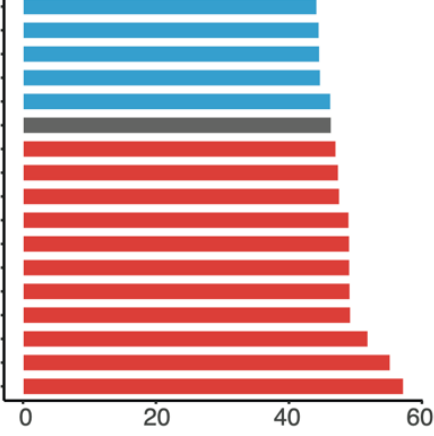

Average percentage of quiescent cells (\%) 
bioRxiv preprint doi: https://doi.org/10.1101/2021.11.12.468410; this version posted December 3, 2021. The copyright holder for this

preprint (which was not certified by peer review) is the author/funder, who has granted bioRxiv a license to display the preprint in perpetuity. It is made available under aCC-BY-NC-ND 4.0 International license.

compounds. Treatments highlighted in blue and red indicate the 30 compounds which showed the

1107 highest decrease and increase in quiescence levels, respectively, in comparison to the control,

1108 shown in grey. (c-d) The average percentage of quiescent MCF7 (c) and A549 (d) cells following a

1109 24-hour treatment with compounds affecting specific pathways. Pathways shown in blue and red

1110 indicate an average decrease and increase in quiescence respectively in comparison to the control,

1111 shown in grey. 
a

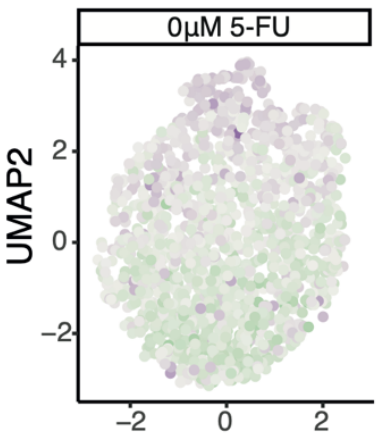

TP53-WT RKO cells
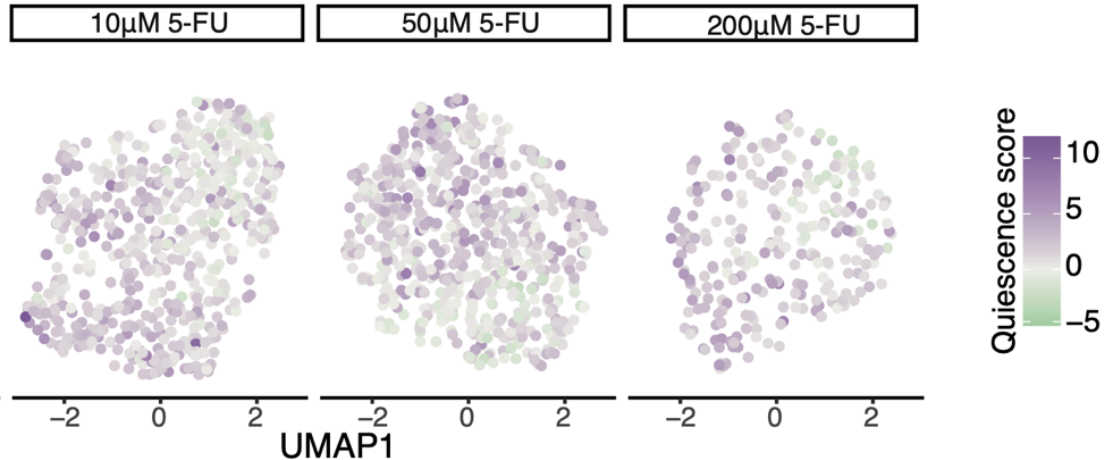

b

TP53-MT SW480 cells
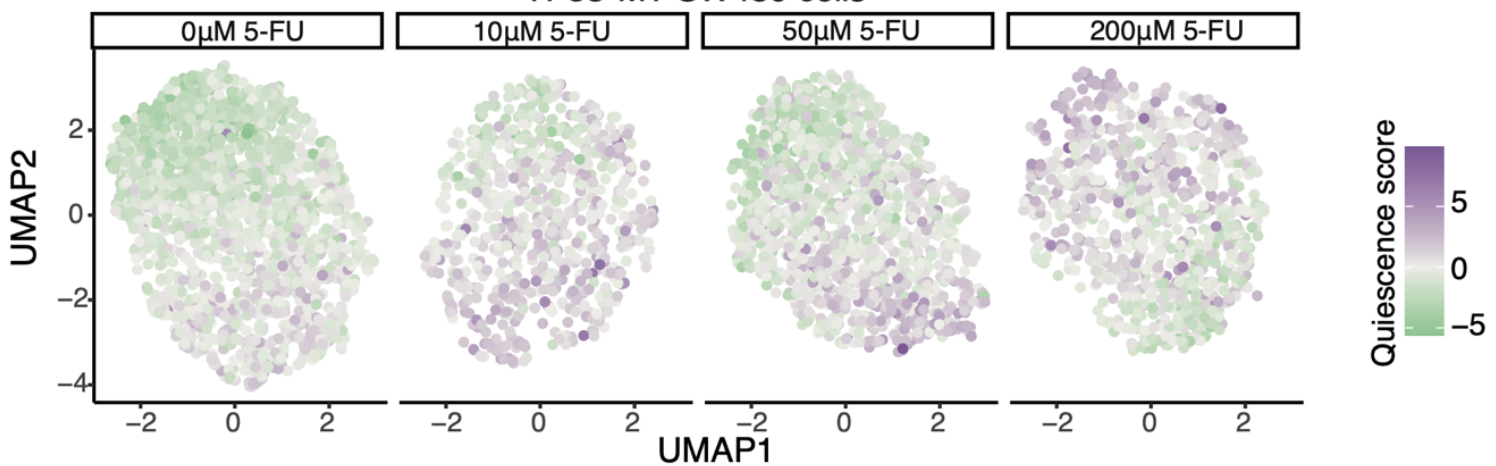

c
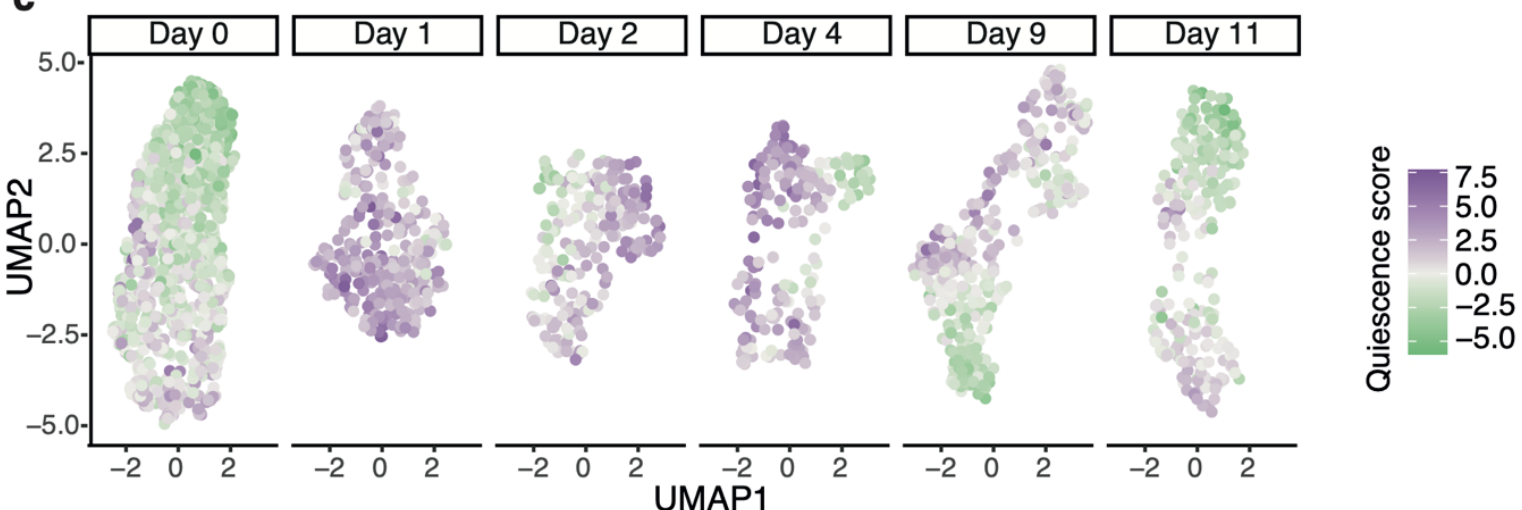

d
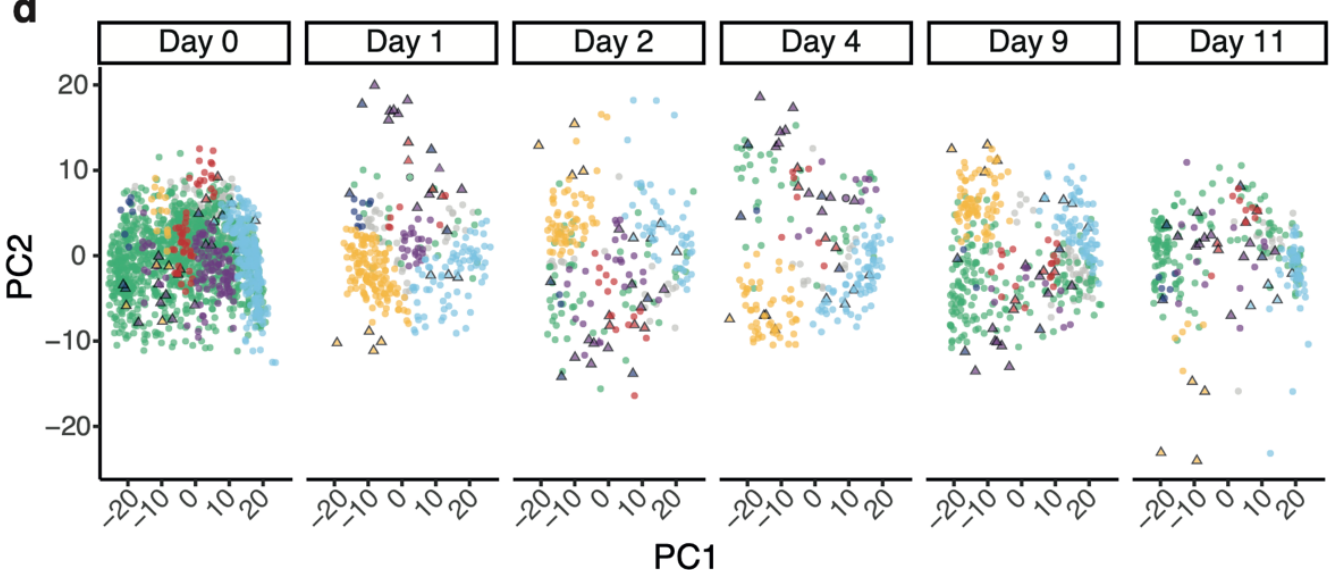

Sample type

- scRNA-seq

$\triangle$ RNA-seq

reference

Quiescence type

- CDK4/6 Inhibition

- Contact Inhibition

- MEK Inhibition

- Serum starvation

- Spontaneous

PC1 
to various 5-FU doses, (b) the TP53-deficient SW480 colorectal cancer cell line to various 5-FU doses, and (c) the P9 NSCLC cell line to the EGFR inhibitor Erlotinib across several timepoints.

1117 Each dot is an individual cell and it is coloured according to its dormancy level, calculated using

1118 the optimised 35 gene signature. (d) Principal component analysis illustrating the superimposition

1119 of scRNA-seq profiles (circles) of quiescent NSCLC cells, inferred using the optimised 35 gene

1120 signature, before and after EGFR inhibition onto the bulk RNA-seq reference data (triangles) for

1121 MCF10A cells occupying various quiescence states. The NSCLC cell line quiescence type was

1122 predicted using a k-nearest neighbour algorithm based on the bulk RNA-seq reference. 